\begin{tabular}{|c|c|}
\hline Title & Projective symmetry group analysis of inelastic light scattering in Kitaev spin balls \\
\hline Author(s) & Kimura, Taku; Y amamoto, Shoji \\
\hline Citation & $\begin{array}{l}\text { Physical Review B, 101(21), } 214411 \\
\text { https://doi.org/10.1103PPysRevB.101.214411 }\end{array}$ \\
\hline Issue Date & 2020-06-05 \\
\hline Doc URL & http:/hdl.handle.net/2115/78948 \\
\hline Rights & @2020 A merican Physical Society \\
\hline Type & article \\
\hline File Information & PhysRevB.101-21_214411.pdf \\
\hline
\end{tabular}

Instructions for use 


\title{
Projective symmetry group analysis of inelastic light scattering in Kitaev spin balls
}

\author{
Taku Kimura and Shoji Yamamoto (1)* \\ Department of Physics, Hokkaido University, Sapporo 060-0810, Japan
}

(Received 3 February 2020; revised manuscript received 8 May 2020; accepted 11 May 2020;

published 5 June 2020)

\begin{abstract}
Projective symmetry groups are applied to Raman observations of the Kitaev quantum spin liquids in spherical lattice geometries realized by Platonic and Archimedean polyhedra. Parton single excitations in Kitaev spin polyhedra are characterized by double-valued irreducible representations of their belonging projective symmetry groups, whereas parton geminate excitations relevant to Raman scattering are decomposed into single-valued irreducible representations of the corresponding point symmetry groups. We combine a standard point symmetry group analysis of the Loudon-Fleury vertices and an elaborate projective symmetry group analysis of itinerant spinons against the ground gauge fields to reveal hidden selection rules for Raman scattering in $\mathbb{Z}_{2}$ spin liquids.
\end{abstract}

DOI: 10.1103/PhysRevB.101.214411

\section{INTRODUCTION}

The Kitaev honeycomb model [1] sparked brand new interest in quantum spin liquids (QSLs) [2-5]. It is exactly solvable to have a QSL ground state accompanied by $\mathbb{Z}_{2}$ gauge fields, whose excitations are fractional, decomposing into itinerant "spinons" and local gapped "visons." Jackeli and Khaliullin [6] designed Mott insulators in the strong spin-orbit coupling limit for the Kitaev model, leading to many candidate materials such as $\mathrm{Na}_{2} \mathrm{IrO}_{3}$ [7], $\alpha$ - $\mathrm{Li}_{2} \mathrm{IrO}_{3}$ [8], $\mathrm{H}_{3} \mathrm{LiIr}_{2} \mathrm{O}_{6}$ [9], and $\alpha-\mathrm{RuCl}_{3}$ [10]. The pure Kitaev model is hard to realize but often accompanied by not only usual Heisenberg interactions, whether intralayer [11,12] or interlayer [13-16], but also off-diagonal exchanges referred to as the $\Gamma$ term [17-19]. Since fractional excitations remain possible in such "effective" Kitaev models [4,5,20-29], inelastic neutron scattering [30-33], x-ray absorption [10], and Raman scattering [34] measurements have been performed on them in an attempt to diagnose QSLs. Raman spectroscopy is particularly useful in detecting spinons separately from visons [24,25,35].

The Kitaev QSL is realizable with any lattice of coordination number 3. $\beta-\mathrm{Li}_{2} \mathrm{IrO}_{3}$ [36] and $\gamma-\mathrm{Li}_{2} \mathrm{IrO}_{3}$ [37], consisting of "hyperhoneycomb" [38,39] and "stripyhoneycomb" [40] lattices, respectively, are such candidates in three dimensions. While they both exhibit gapless spinon excitations coming from nodal rings, the degeneracy of the Fermi level strongly depends on the lattice geometry in general. A normal Fermi surface is emergent in a "hyperoctagon" lattice [41,42], whereas it reduces to what are called Weyl points in "hypernonagon" $[42,43]$ and "hyperhexagon" $[42,44]$ lattices. Spinon excitations may be gapped from the ground state [42]. Kitaev models of lower than two dimensions also attract much interest. Kitaev honeycomb nanoribbons with both zigzag and armchair edges are discussed in an attempt to optically distinguish between different topological phases [45] and investigated with particular interest in a bulk-edge correspondence

\footnotetext{
*yamamoto@phys.sci.hokudai.ac.jp
}

[46], i.e., a possible relation between gapped states in the bulk and gapless states in the boundary. A Kitaev spin ladder maps onto a one-dimensional $p$-wave superconductor in terms of Dirac fermions to reveal the equivalence between spontaneous global $\mathbb{Z}_{2}$ symmetry breaking and emergent isolated Majorana modes [47], while that with inhomogeneous exchange interactions exhibits coexistent different topological phases with Majorana end states in between [48].

In such circumstances, Mellado, Petrova, and Tchernyshyov (MPT) [49] discuss the Kitaev spin model in a spherical lattice geometry realized by Archimedean solids. Analyzing the projective symmetry [50,51] of the gaugeground Majorana fermionic Hamiltonian (cf. Appendix A) rather than the point symmetry of the background lattice, they claim that a parton behaves like an electrically charged particle in a radial (monopole) magnetic field within the continuum - in the sense of a perfect sphere-approximation. This parton has a half-odd-integral orbital angular momentum due to the magnetic monopole located at the center of the cluster.

Motivated by the MPT theory, we present a symmetry argument of optical observations of "Kitaev spin balls", i.e., QSLs in a spherical lattice geometry realized by Platonic and Archimedean polyhedra (cf. Fig. 1). Since Raman scattering within the Loudon-Fleury (LF) scheme [53-55] is mediated by spinons in pairs, we make direct-product representations out of irreducible representations of the corresponding projective symmetry group and then decompose them into irreducible representations again. In order to reveal how each spinon geminate excitation behaves under spatial inversion, which is vitally important in the context of Raman scattering, we go so far as to take gauged inversion, if any, as well as gauged rotations, into the projective symmetry. Kitaev spin balls made only of $2 l$-sided polygons $(l \in \mathbb{N})$ require such an elaborate formulation, namely, making direct-product representations of the extended binary polyhedral group, i.e., the double cover of the full icosahedral or octahedral group, to obtain inversion-symmetry-definite single-valued irreducible representations. 


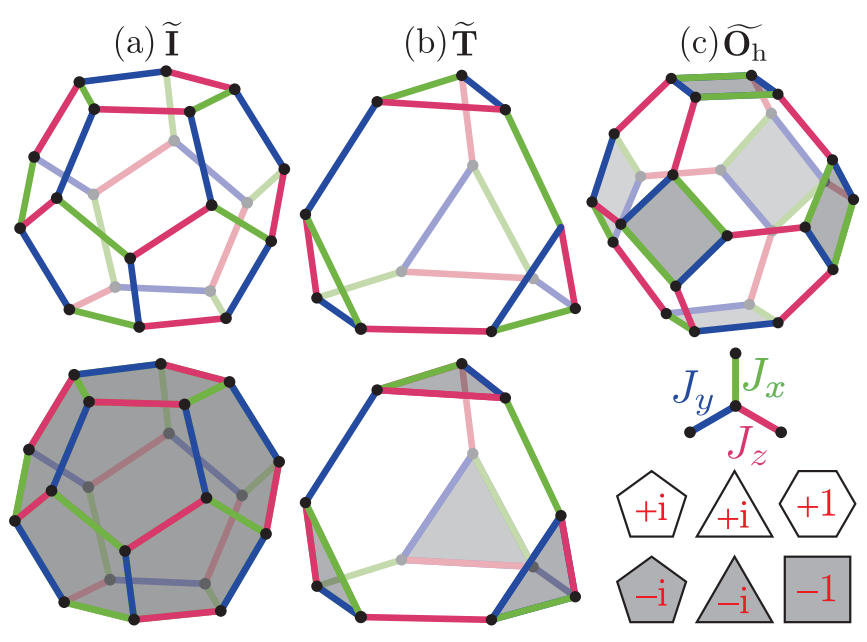

FIG. 1. Kitaev spin balls consisting of (a) dodecahedral, (b) truncated-tetrahedral, and (c) truncated-octahedral lattices in their ground flux configurations. The ground state of the truncated octahedron is unique, whereas those of the dodecahedron and truncated tetrahedron are both degenerate [52], with their constituent pentagons arrangeable into either $\left\{W_{p}=+i ; p=1, \ldots, 12\right\}$ or $\left\{W_{p}=-i ; p=1, \ldots, 12\right\}$ and triangles arrangeable into either $\left\{W_{p}=+i ; p=1, \ldots, 4\right\}$ or $\left\{W_{p}=-i ; p=1, \ldots, 4\right\}$.

\section{KITAEV MODELS OF PLATONIC AND ARCHIMEDEAN POLYHEDRA}

The Kitaev Hamiltonian (Fig. 1) reads

$$
\mathscr{H}=-\sum_{\lambda=x, y, z} \sum_{\langle m, n\rangle_{\lambda}} J_{\lambda} \sigma_{m}^{\lambda} \sigma_{n}^{\lambda},
$$

where $\left(\sigma_{l}^{x}, \sigma_{l}^{y}, \sigma_{l}^{z}\right)(l=1, \ldots, L)$ are the Pauli matrices and $\langle m, n\rangle_{\lambda}(\lambda=x, y, z)$ each run over a different set of $L / 2$ nearest-neighbor bonds between the $\lambda$ components. We set this model in various polyhedral geometries, i.e., on dodecahedral, truncated-tetrahedral, and truncated-octahedral lattices, whose point symmetry groups are given by $\mathbf{I}_{\mathrm{h}}=\mathbf{I} \times \mathbf{C}_{\mathrm{i}}$, $\mathbf{T}_{\mathrm{d}}=\mathbf{T}+I C_{4} \mathbf{T}$, and $\mathbf{O}_{\mathrm{h}}=\mathbf{O} \times \mathbf{C}_{\mathrm{i}}=\mathbf{T}_{\mathrm{d}} \times \mathbf{C}_{\mathrm{i}}$, respectively. $J_{x}, J_{y}$, and $J_{z}$ are all set to $J>0$ in the following.

By representing the spin operators in terms of four Majorana fermions, $\sigma_{l}^{\lambda}=i \eta_{l}^{\lambda} c_{l}$, with anticommutation relations between them, $\left\{\eta_{m}^{\mu}, \eta_{n}^{\nu}\right\}=2 \delta_{m n} \delta_{\mu \nu},\left\{c_{m}, c_{n}\right\}=2 \delta_{m n}$, and $\left\{\eta_{m}^{\lambda}, c_{n}\right\}=0$, and then introducing bond operators, $\hat{u}_{\langle m, n\rangle_{\lambda}} \equiv$ $i \eta_{m}^{\lambda} \eta_{n}^{\lambda}$, the spin Hamiltonian (1) is rewritten as

$$
\mathscr{H}=i J \sum_{\lambda=x, y, z} \sum_{\langle m, n\rangle_{\lambda}} \hat{u}_{\langle m, n\rangle_{\lambda}} c_{m} c_{n} .
$$

Since $\left[\hat{u}_{\langle m, n\rangle_{\lambda}}, \mathscr{H}\right]=0$ and $\hat{u}_{\langle m, n\rangle_{\lambda}}^{2}=1, \hat{u}_{\langle m, n\rangle_{\lambda}}$ reads as a $\mathbb{Z}_{2}$ classical variable, $u_{\langle m, n\rangle_{\lambda}}= \pm 1$. Numbering the constituent polygons of a polyhedra, $p=1, \ldots, \frac{L}{2}+2$, we define a flux operator $[1,56]$ for each by multiplying its $N_{p}$ spin operators in the anticlockwise manner viewed from the outside of the polyhedron,

$$
\begin{aligned}
\hat{W}_{p} & \equiv e^{i \hat{\Phi}_{p}}=\prod_{\langle m, n\rangle_{\lambda} \in \partial p} \sigma_{m}^{\lambda} \sigma_{n}^{\lambda} \\
& =(-i)^{N_{p}} \prod_{\langle m, n\rangle_{\lambda} \in \partial p} \hat{u}_{\langle m, n\rangle_{\lambda}} .
\end{aligned}
$$

$\hat{W}_{p}$ also commutes with (2) and thus behaves as a classical variable, $W_{p}= \pm 1$ or $\pm i$, according to whether $N_{p}$ is even or odd. A U(1) gauge flux, $W_{p} \equiv e^{i \Phi_{p}}\left(-\pi<\Phi_{p} \leqslant \pi\right)$, pierces the constituent polygon $p$. Every Kitaev spin ball consists of $\frac{L}{2}+2$ gauged polygons with their flux variables satisfying $\prod_{p=1}^{\frac{L}{2}+2} W_{p}=1$. The Hilbert space of the spin Hamiltonian (1) is block-diagonal with respect to flux configurations $\left\{W_{p}\right\}$, consisting of $2^{\frac{L}{2}+1}$ blocks of dimension $2^{\frac{L}{2}-1} \times 2^{\frac{L}{2}-1}$, while that of the augmented Majorana Hamiltonian (2) is blockdiagonal with respect to bond configurations $\left\{u_{\langle m, n\rangle_{\lambda}}\right\}$ as well as $\left\{W_{p}\right\}$, consisting of $2^{\frac{3 L}{2}}$ blocks of dimension $2^{\frac{L}{2}} \times 2^{\frac{L}{2}}$. Four Majorana fermions at each site have $2^{2 L}$ degrees of freedom, containing "unphysical states" $[57,58]$ to be projected out by the operator [57-60]

$$
\mathscr{P}=\prod_{l=1}^{L} \frac{1}{2}\left(1+\eta_{l}^{x} \eta_{l}^{y} \eta_{l}^{z} c_{l}\right) .
$$

Once a set of the $3 L / 2$ gauge fields $\left\{u_{\langle m, n\rangle_{\lambda}}\right\}$ is given, we have a Majorana quadratic Hamiltonian to be solved:

$$
\begin{aligned}
\mathscr{H} & =\frac{i}{2} \sum_{m=1}^{L} \sum_{n=1}^{L} \mathcal{H}_{m n} c_{m} c_{n} ; \\
\mathcal{H}_{m n} & =-\mathcal{H}_{n m} \equiv J u_{\langle m, n\rangle_{\lambda}} .
\end{aligned}
$$

The real skew-symmetric matrix $\mathcal{H}$ can be block-diagonalized by a real orthogonal matrix $\boldsymbol{\Psi}$ :

$$
\begin{aligned}
& \mathscr{H}=\frac{i}{2}{ }^{t} \boldsymbol{\Psi} \boldsymbol{\Psi}^{\mathrm{t}} \boldsymbol{\Psi} \mathcal{H} \boldsymbol{\Psi}^{\mathrm{t}} \boldsymbol{\Psi} \boldsymbol{c}=\frac{i}{2} \tilde{\boldsymbol{c}} \mathcal{E} \tilde{\boldsymbol{c}}=i \sum_{k=1}^{L / 2} \frac{\varepsilon_{k}}{2} \tilde{c}_{2 k-1} \tilde{c}_{2 k}, \\
& \boldsymbol{c} \equiv\left[\begin{array}{c}
c_{1} \\
\vdots \\
c_{L}
\end{array}\right]=\left[\begin{array}{ccc}
\psi_{1,1} & \cdots & \psi_{1, L} \\
\vdots & \ddots & \vdots \\
\psi_{L, 1} & \cdots & \psi_{L, L}
\end{array}\right]\left[\begin{array}{c}
\tilde{c}_{1} \\
\vdots \\
\tilde{c}_{L}
\end{array}\right] \equiv \boldsymbol{\Psi} \tilde{\boldsymbol{c}} \\
& \tilde{\boldsymbol{c}}={ }^{\mathrm{t}} \boldsymbol{\Psi} \boldsymbol{c}, \mathcal{E} \equiv \frac{1}{2}\left[\begin{array}{ccccc}
0 & \varepsilon_{1} & & & \\
-\varepsilon_{1} & 0 & & & \\
& & \ddots & & \\
& & & 0 & \varepsilon_{\frac{L}{2}} \\
& & & -\varepsilon_{\frac{L}{2}} & 0
\end{array}\right]
\end{aligned}
$$

We recomplexify Majorana fermions,

$$
\begin{aligned}
\tilde{c}_{2 k-1} & =\alpha_{k}^{\dagger}+\alpha_{k}, \quad \tilde{c}_{2 k}=i\left(\alpha_{k}^{\dagger}-\alpha_{k}\right), \\
c_{l} & =\sum_{k=1}^{L / 2}\left(\psi_{l, 2 k-1} \tilde{c}_{2 k-1}+\psi_{l, 2 k} \tilde{c}_{2 k}\right) \\
& =\sum_{k=1}^{L / 2}\left[\left(\psi_{l, 2 k-1}+i \psi_{l, 2 k}\right) \alpha_{k}^{\dagger}+\left(\psi_{l, 2 k-1}-i \psi_{l, 2 k}\right) \alpha_{k}\right], \\
\alpha_{k} & =\frac{1}{2}\left(\tilde{c}_{2 k-1}+i \tilde{c}_{2 k}\right)=\frac{1}{2} \sum_{l=1}^{L}\left(\psi_{l, 2 k-1}+i \psi_{l, 2 k}\right) c_{l}, \\
\alpha_{k}^{\dagger} & =\frac{1}{2}\left(\tilde{c}_{2 k-1}-i \tilde{c}_{2 k}\right)=\frac{1}{2} \sum_{l=1}^{L}\left(\psi_{l, 2 k-1}-i \psi_{l, 2 k}\right) c_{l},
\end{aligned}
$$


so as to obtain a diagonal Hamiltonian,

$$
\mathscr{H}=\sum_{k=1}^{L / 2} \frac{\varepsilon_{k}}{2}\left(\alpha_{k}^{\dagger} \alpha_{k}-\alpha_{k} \alpha_{k}^{\dagger}\right)=\sum_{k=1}^{L / 2} \varepsilon_{k}\left(\alpha_{k}^{\dagger} \alpha_{k}-\frac{1}{2}\right),
$$

with nonnegative eigenvalues $\varepsilon_{k} \geqslant 0$. Note that all sets of the gauge fields $\left\{u_{\langle m, n\rangle_{\lambda}} ;\langle m, n\rangle_{x},\langle m, n\rangle_{y},\langle m, n\rangle_{z}=1, \ldots, \frac{L}{2}\right\}$ yielding the same flux configuration $\left\{W_{p} ; p=1, \ldots, \frac{L}{2}+2\right\}$ give the same set of eigenvalues $\left\{\varepsilon_{k} ; k=1, \ldots, \frac{L}{2}\right\}$. $\mathscr{P}$ can be expressed in terms of the bond variables $u_{\langle m, n\rangle_{\lambda}}$, mixing coefficients $\psi_{l, l^{\prime}}$, and quasiparticle occupation operators $\alpha_{k}^{\dagger} \alpha_{k}$ to act on quasiparticle (spinon) states labeled background gauge fields $\left\{u_{\langle m, n\rangle_{\lambda}}\right\}$. Physical (unphysical) spinon states in the ground (lowest-energy) gauge sector consist of even (odd) numbers of emergent spinons $\alpha_{k}^{\dagger} \alpha_{k}$. All the $2^{\frac{3 L}{2}}$ gauge sectors each contain $2^{\frac{L}{2}-1}$ physical and $2^{\frac{L}{2}-1}$ unphysical states, each consisting of either only even or only odd numbers of spinons.

The ground flux configurations of Kitaev spin balls (Fig. 1) are such that $W_{p}$ of every constituent $N_{p}$-sided polygon is +1 , -1 , or either of $+i$ and $-i$ according to whether $N_{p}$ is $4 l+2$, $4 l$, or $2 l+1$ with $l \in \mathbb{N}[49,56]$. With the time-reversalinvariant Hamiltonian, the ground state is at least doubly degenerate unless all $N_{p}$ 's are even [52]. Considering that the eigenspectrum of (2) depends on $\left\{u_{\langle m, n\rangle_{\lambda}}\right\}$ only through $\left\{W_{p}\right\}$ and the $W_{p}$ 's each commute with (1) as well as (2), we describe the ground state, unless otherwise noted, as a spinon vacuum against a ground flux configuration,

$$
\left|\left\{n_{k}\right\}\right\rangle_{0} \otimes\left|\left\{W_{p}\right\}\right\rangle_{0} \equiv|0\rangle
$$

where we denote the $\kappa$ th spinon state against the $q$ th flux configuration by $\left|\left\{n_{k}\right\}\right\rangle_{\kappa} \otimes\left|\left\{W_{p}\right\}\right\rangle_{q}\left(\kappa=0, \ldots, 2^{\frac{L}{2}-1}-1 ; q=\right.$ $\left.0, \ldots, 2^{\frac{L}{2}+1}-1\right)$, allowing it to run over physical states only.

\section{PROJECTIVE SYMMETRY GROUPS FOR GAUGE-GROUND KITAEV POLYHEDRA}

\section{A. Single- and double-valued irreducible representations}

Characterizing Raman scattering mediated by Majorana spinons emergent in the gauge-ground Kitaev truncated octahedron in terms of its projective symmetry group is essentially twofold: first, we go further than MPT [49] in obtaining a projective symmetry group for single Majorana eigenmodes, i.e., construct the double cover of the $\mathrm{O}(3)$ superset of a pure rotation group, and then analyze direct-product representations made of its double-valued irreducible representations. Let us denote the point symmetry group of a Kitaev spin ball and its arbitrary group element by $\mathbf{P}$ and $P$, respectively, and the $\mathbb{Z}_{2}$-gauge extension of $\mathbf{P}$ and resultant gauged point symmetry operations by $\widetilde{\mathbf{P}}$ and $\widetilde{P}$, respectively. Regular and semiregular polyhedral lattices of our interest have the same coordination number 3 and their point symmetry groups are either the cubic $\left(\mathbf{T}_{\mathrm{d}}, \mathbf{O}_{\mathrm{h}}\right)$ or the icosahedral $\left(\mathbf{I}_{\mathrm{h}}\right)$ groups. Therefore, $\mathbf{P} \subset \mathrm{O}(3)$ in general. $P \in \mathbf{P}$ generally changes the ground gauge fields of the Majorana Hamiltonian. We demonstrate in detail gauged point symmetry operations on gauge-ground Kitaev polyhedra as well as pure point symmetry operations on their background lattices in Appendix A. Any two bond configurations yielding the same set of fluxes can be converted to each other by local gauge operations. Every rotation
$R \in \mathbf{R}$ leaves any flux configuration $\left\{W_{p} ; p=1, \ldots, \frac{L}{2}+2\right\}$ unchanged, whereas the inversion $I \in \mathbf{P}$ and every reflection $\sigma \in \mathbf{P}$ reverse the signs of all imaginary $W_{p}$ 's peculiar to polygons of odd $N_{p}$. Only if the group action $P$ leaves the flux configuration $\left\{W_{p}\right\}$ unchanged does there exist a pair of gauge transformations $\pm \Lambda(P)$ to recover the initial ground gauge fields, $\pm \Lambda(P) P\left\{u_{\langle m, n\rangle_{\lambda}}\right\}=\left\{u_{\langle m, n\rangle_{\lambda}}\right\}$. We denote a couple of gauged point symmetry operations $\pm \Lambda(P) P$ unifiedly as $\widetilde{P}$ and distinguishably by $\bar{P}$ and $\underline{P}$. The symmetry groups of the gauge-ground Kitaev dodecahedron and truncated tetrahedron are the $\mathbb{Z}_{2}$-gauge extensions of the $\mathrm{SO}(3)$ subgroups of their full point symmetry groups, $\widetilde{\mathbf{I}}$ and $\widetilde{\mathbf{T}}$, respectively, whereas that of the gauge-ground Kitaev truncated octahedron is the $\mathbb{Z}_{2}$-gauge extension of its full point symmetry group, $\widetilde{\mathbf{O}_{\mathrm{h}}}$. While gauged rotations $\widetilde{R}$ with $R \in \mathbf{O}$ and gauged inversions $\widetilde{I}$ with $I \in \mathbf{C}_{\mathrm{i}}$ are all symmetry operations of the gaugeground Kitaev truncated octahedron, they are not necessarily commutable because every gauge transformation $\Lambda(P)$ is obedient to the preceding point symmetry operation $P$. All the $g^{\widetilde{\mathbf{o}}} \times g^{\widetilde{\mathbf{C}}_{\mathrm{i}}}+g^{\widetilde{\mathbf{C}}_{\mathrm{i}}} \times g^{\widetilde{\mathbf{o}}}=384$ products between the $g^{\widetilde{\mathbf{o}}}$ elements of $\widetilde{\mathbf{O}}$ and the $g \widetilde{\mathbf{C}}_{i}$ elements of $\widetilde{\mathbf{C}}_{\mathrm{i}}$ are indeed symmetry operations of the gauge-ground Kitaev truncated octahedron, but they quadruply count the $g^{\widetilde{\mathbf{O}_{\mathrm{h}}}}=96$ elements of $\widetilde{\mathbf{O}_{\mathrm{h}}}=$ $\widetilde{\mathbf{O}}+\bar{I} \widetilde{\mathbf{O}}$. Note further that the symmetry group of the gaugeground Kitaev truncated octahedron is different from that of half-integral spins in an octahedral environment, $\widetilde{\mathbf{O}} \times \mathbf{C}_{\mathrm{i}}(\mathrm{cf}$. Appendix B), where $\widetilde{\mathbf{O}} \subset \mathrm{SU}(2)$, being a double covering group for the pure rotation group $\mathbf{O} \subset \mathrm{SO}(3)$, commutes with $\mathbf{C}_{\mathrm{i}}$ because inversion has no effect on any angular momentum [61].

We are now in a position to construct the double group $\widetilde{\mathbf{O}_{\mathrm{h}}}$. The 48 elements of $\mathbf{O}_{\mathrm{h}}$ can be divided into 10 classes: $\{E\}$, $\left\{6 C_{4}\right\},\left\{3 C_{2}\right\},\left\{6 C_{2}^{\prime}\right\},\left\{8 C_{3}\right\},\{I\},\left\{6 I C_{4}\right\},\left\{3 I C_{2}\right\},\left\{6 I C_{2}^{\prime}\right\},\left\{8 I C_{3}\right\}$. The 96 elements of $\widetilde{\mathbf{O}_{\mathrm{h}}}$ can be divided into 13 classes: $\{\bar{E}\}$, $\{\underline{E}\},\left\{6 \overline{C_{4}}, 6 \underline{C_{4}}\right\},\left\{3 \overline{C_{2}}, 3 \underline{C_{2}}\right\},\left\{6 \overline{C_{2}^{\prime}}, 6 \underline{C_{2}^{\prime}}\right\},\left\{8 \overline{C_{3}}\right\},\left\{8 \underline{C_{3}}\right\},\{\bar{I}, \underline{I}\}$, $\left\{6 \overline{I C_{4}}, 6 I C_{4}\right\},\left\{3 \overline{I C_{2}}, 3 I \overline{C_{2}}\right\},\left\{6 \overline{I C_{2}^{\prime}}, 6 \overline{I C_{2}^{\prime}}\right\},\left\{8 \overline{I C_{3}}\right\},\left\{8 I C_{3}\right\}$. The

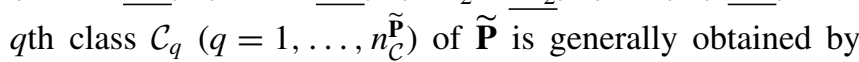
gauging point symmetry operations of the same type to yield $h_{q}$ elements in such ways that $\left\{h_{q} \bar{P}_{q}\right\},\left\{h_{q} \underline{P}_{q}\right\}$, or $\left\{\frac{h_{q}}{2} \bar{P}_{q}, \frac{h_{q}}{2} \underline{P}_{q}\right\}$. Let us denote the $i$ th irreducible representation of $\mathbf{P}(\widetilde{\mathbf{P}})$ by $\Xi_{i}$ $\left(\widetilde{\Xi}_{i}\right)$ and its dimensionality by $d_{\Xi_{i}}^{\mathbf{P}}\left(d_{\widetilde{\Xi}_{i}}\right)$. Having in mind that all the single-valued irreducible representations of $\mathbf{O}_{\mathrm{h}}$ remain unchanged in $\widetilde{\mathbf{O}}_{\mathrm{h}}, \widetilde{\Xi}_{i}=\Xi_{i}(i=1, \ldots, 10)$, we compare

$$
\sum_{i=1}^{n_{C}^{\mathbf{O}_{\mathrm{h}}} \equiv 10}\left(d_{\Xi_{i}}^{\mathbf{O}_{\mathrm{h}}}\right)^{2}=g^{\mathbf{O}_{\mathrm{h}}}, \quad \sum_{i=1}^{n_{C}^{\widetilde{O_{\mathrm{h}}}} \equiv 13}\left(d_{\widetilde{\Xi}_{i}}^{\widetilde{\widetilde{O}_{\mathrm{h}}}}\right)^{2}=g^{\widetilde{\mathbf{O}_{\mathrm{h}}}}
$$

to reveal that the three double-valued irreducible represen-

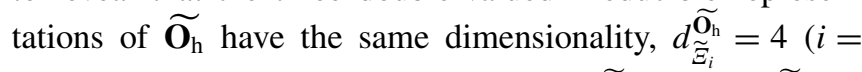
$11,12,13)$. Since their characters satisfy $\chi_{\widetilde{\Xi}_{i}}^{\widetilde{O_{\mathrm{B}}}}(\bar{P})=-\chi_{\widetilde{\Xi}_{i}}(\underline{P})$, we readily find that $\chi_{\widetilde{\Xi}_{i}}^{{\widetilde{O_{\mathrm{h}}}}_{i}}(\bar{E})=-\chi_{\widetilde{\Xi}_{i}}{\widetilde{\sigma_{\mathrm{h}}}}_{(E)}(\underline{E})=4$ and $\chi_{\widetilde{\Xi}_{i}} \underset{\widetilde{\Xi}_{\mathrm{h}}}{\widetilde{\Xi}_{i}}(\widetilde{P})=$ $0\left(P=C_{4}, C_{2}, C_{2}^{\prime}, I, I C_{4}, I C_{2}, I C_{2}^{\prime}\right)$, while the rest $\chi_{\widetilde{\Xi}_{i}} \widetilde{P}_{\bar{P}}(\widetilde{P})$ $\left(P=C_{3}, I C_{3}\right)$ are obtainable through the first orthogonality 


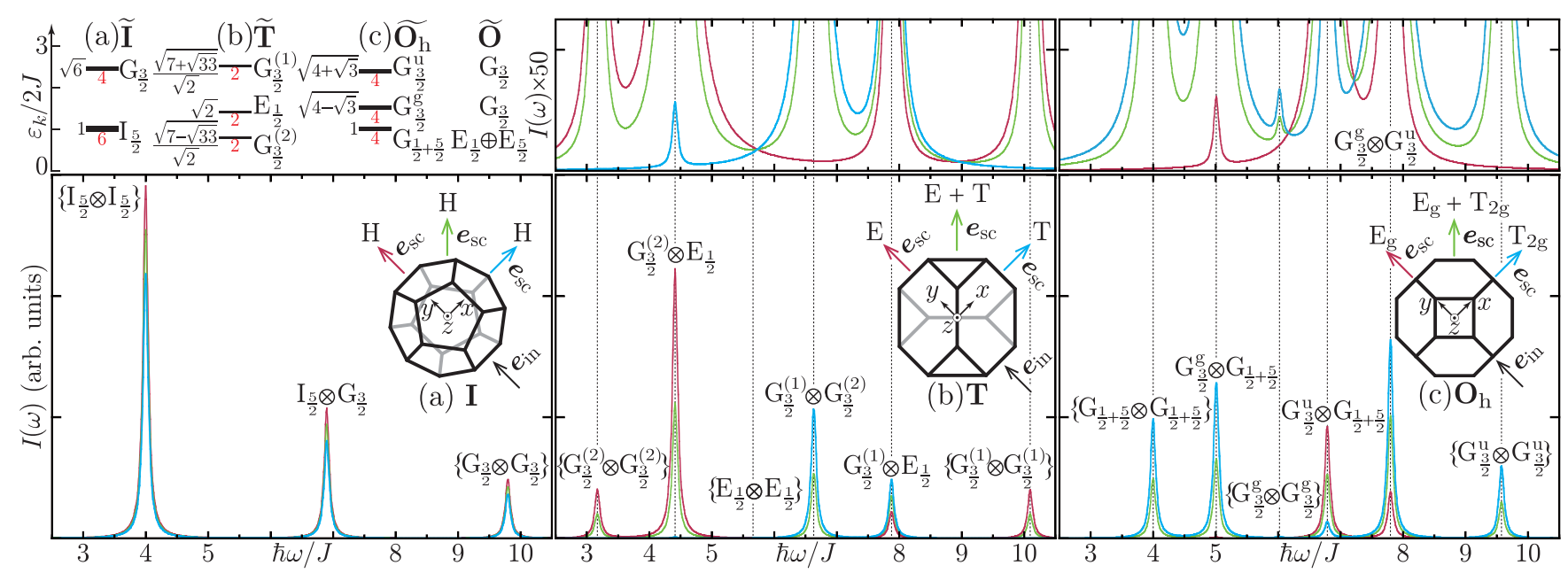

FIG. 2. Spinon excitation energies $\varepsilon_{k}$ and Raman intensities $I(\omega)$ of Kitaev spin balls consisting of (a) dodecahedral, (b) truncatedtetrahedral, and (c) truncated-octahedral lattices in their ground flux configurations, where $\delta$-function peaks are Lorentzian-broadened by $0.05 J$ [59]. The eigenenergy, multiplicity, and irreducible representation are specified beside each eigenlevel. For the incident polarization $\left(\frac{\pi}{2}, \frac{\pi}{2}\right)$, we observe various scattered polarizations $\left(\frac{\pi}{2}, \frac{l \pi}{4}\right)(l=0,1,2)$, each consisting of peaks attributable to direct-product representations

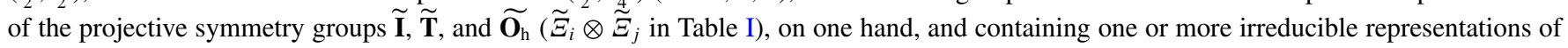
the point symmetry groups $\mathbf{I}, \mathbf{T}$, and $\mathbf{O}_{\mathrm{h}}\left(\bigoplus_{k} \Xi_{k}\right.$ in Table I), on the other hand.

relation (cf. Appendix B)

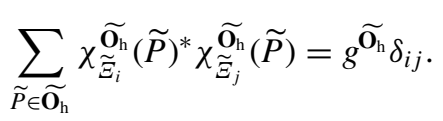

We name the thus-obtained double-valued irreducible representations $G_{\frac{3}{2}}^{\mathrm{g}}, G_{\frac{3}{2}}^{\mathrm{u}}$, and $\mathrm{G}_{\frac{1}{2}+\frac{5}{2}}$ so that they signify the geradelike or ungeradelike response to a gauged point symmetry operation as well as suggest the compatibility relations between $\widetilde{\mathbf{O}_{\mathrm{h}}}$ and its subgroup $\widetilde{\mathbf{O}}, \mathrm{G}_{\frac{1}{2}+\frac{5}{2}} \downarrow \widetilde{\mathbf{O}}=\mathrm{E}_{\frac{1}{2}} \oplus \mathrm{E}_{\frac{5}{2}}$ and $\mathrm{G}_{\frac{3}{2}}^{\mathrm{g}} \downarrow \widetilde{\mathbf{O}}=\mathrm{G}_{\frac{3}{2}}^{\mathrm{u}} \downarrow \widetilde{\mathbf{O}}=\mathrm{G}_{\frac{3}{2}}$, i.e.,

$$
\begin{aligned}
& \chi_{\mathrm{G}_{\frac{1}{2}+\frac{5}{2}}}^{\widetilde{\mathrm{O}_{\mathrm{h}}}}(\widetilde{P})=\chi_{\mathrm{E}_{\frac{1}{2}}}^{\widetilde{\widetilde{P}}}(\widetilde{P})+\chi_{\mathrm{E}_{\frac{5}{2}}}^{\widetilde{\mathbf{O}^{2}}}(\widetilde{P}),
\end{aligned}
$$

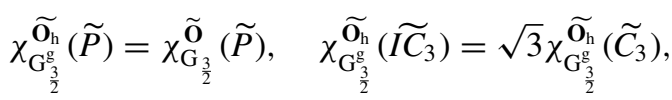

$$
\begin{aligned}
& \chi_{\mathrm{G}_{\frac{3}{2}}^{u}}^{\widetilde{\sigma_{\mathrm{h}}}}(\widetilde{P})=\chi_{\mathrm{G}_{\frac{3}{2}}}^{\widetilde{\widetilde{P}}}(\widetilde{P}), \quad \chi_{\mathrm{G}_{\frac{3}{2}}^{\mathrm{u}}}^{\widetilde{\sigma_{\mathrm{h}}}}\left(\widetilde{I C_{3}}\right)=-\sqrt{3} \chi_{\mathrm{G}_{\frac{3}{2}}^{u}}^{\widetilde{\widetilde{C}_{\mathrm{h}}}}\left(\widetilde{C_{3}}\right) .
\end{aligned}
$$

The Majorana spinon spectrum of the gauge-ground $\widetilde{\mathbf{O}_{\mathrm{h}}} \mathbf{K i}-$ taev polyhedron thus consists of three quadruplets $3 \times 4=$ $L / 2$ [see Fig. 2 together with Eq. (8)]. If we employ $\widetilde{\mathbf{O}}$ [49] in this context, we have two doublets, $\mathrm{E}_{\frac{1}{2}}$ and $\mathrm{E}_{\frac{5}{2}}$, instead of the quadruplet $\mathrm{G}_{\frac{1}{2}+\frac{5}{2}}$, and they look accidentally degenerate with each other. Only the full symmetry group $\widetilde{\mathbf{O}_{\mathrm{h}}}$ can reveal the necessary quadruplet. All the $L / 2$ Majorana spinon eigenmodes of the gauge-ground $\widetilde{\mathbf{I}}$ and $\widetilde{\mathbf{T}}$ Kitaev polyhedra are also describable with double-valued irreducible representations of their projective symmetry groups [see Fig. 2 together with Eq. (8)]. The former consist of a sextuplet of $I_{\frac{5}{2}}$ and a quadruplet of $\mathrm{G}_{\frac{3}{2}}$, while the latter consist of three doublets of $\mathrm{G}_{\frac{3}{2}}^{(1)}, \mathrm{G}_{\frac{3}{2}}^{(2)}$, and $\mathrm{E}_{\frac{1}{2}}$, where the four-dimensional real irreducible representation $\mathrm{G}_{\frac{3}{2}}$ splits into the two-dimensional complex ones $\mathrm{G}_{\frac{3}{2}}^{(1)}$ and $\mathrm{G}_{\frac{3}{2}}^{(2)}$ due to the pure imaginary Hamiltonian (8). Irreducible representations of the double groups $\widetilde{\mathbf{I}}, \widetilde{\mathbf{T}}, \widetilde{\mathbf{O}}$, and $\widetilde{\mathbf{O}_{\mathrm{h}}}$ are analyzed in further detail and listed with their characters in Appendix B.

\section{B. Direct-product representations}

Direct-product representations of a nonabelian group are not necessarily irreducible even though they are made of irreducible representations. Those of the projective symmetry group $\widetilde{\mathbf{P}}$ are generally decomposed into irreducible representations of $\widetilde{\mathbf{P}}$ :

$$
\begin{aligned}
\widetilde{\Xi}_{i} \otimes \widetilde{\Xi}_{j} & =\frac{1}{g^{\widetilde{P}}} \bigoplus_{k=1}^{n_{\mathcal{C}}^{\widetilde{P}}} \widetilde{\Xi}_{k} \sum_{q=1}^{n_{\mathcal{C}}^{\widetilde{P}}} h_{q} \chi_{\widetilde{\Xi}_{k}}^{\widetilde{\widetilde{P}}}\left(\mathcal{C}_{q}\right)^{*} \chi \underset{\widetilde{\Xi}_{i} \otimes \widetilde{\Xi}_{j}}{\widetilde{\mathbb{P}}}\left(\mathcal{C}_{q}\right) ; \\
\chi \widetilde{\Xi}_{i} \otimes \widetilde{\Xi}_{j} & (\widetilde{P})=\chi \chi_{\widetilde{\Xi}_{i}}^{\widetilde{P}}(\widetilde{P}) \chi \chi_{\widetilde{\Xi}_{j}}^{\widetilde{P}}(\widetilde{P}) .
\end{aligned}
$$

Two-spinon-mediated Raman scatterings in a Kitaev QSL are generally labeled with direct-product representations of its projective symmetry group $\mathbf{P}, \widetilde{\Xi}_{i} \otimes \widetilde{\Xi}_{j}\left(i, j=n_{\mathcal{C}}^{\mathbf{P}}+\right.$ $1, \ldots, n_{\mathcal{C}} \mathbf{\mathbb { P }}$, each decomposable into single-valued irreducible representations of the corresponding point symmetry group $\mathbf{P}$,

$$
\widetilde{\Xi}_{i} \otimes \widetilde{\Xi}_{j}=\bigoplus_{k=1}^{n_{\mathcal{C}}^{\mathbf{P}}} \Xi_{k} \sum_{q=1}^{n_{\mathcal{C}}^{\tilde{P}}} \frac{h_{q}}{g^{\widetilde{\mathbf{P}}}} \chi_{\Xi_{k}}^{\mathbf{P}}\left(\mathcal{C}_{q}\right)^{*} \chi_{\widetilde{\Xi}_{i} \otimes \widetilde{\Xi}_{j}}^{\widetilde{\mathbf{P}}}\left(\mathcal{C}_{q}\right),
$$

keeping in mind that

$$
\chi_{\widetilde{\Xi}_{i} \otimes \widetilde{\Xi}_{j}}^{\widetilde{\widetilde{P}}}(\bar{P})=\chi_{\widetilde{\Xi}_{i} \otimes \widetilde{\Xi}_{j}}^{\widetilde{\widetilde{P}}}(\underline{P}) \quad\left(i, j=n_{\mathcal{C}}^{\mathbf{P}}+1, \ldots, n_{\mathcal{C}}^{\widetilde{\mathbf{P}}}\right) .
$$

Direct-product representations made of the two same irreducible representations further decompose into symmetric and 
antisymmetric direct-product representations,

$$
\begin{aligned}
\widetilde{\Xi}_{i} \otimes \widetilde{\Xi}_{i} & =\left[\widetilde{\Xi}_{i} \otimes \widetilde{\Xi}_{i}\right] \oplus\left\{\widetilde{\Xi}_{i} \otimes \widetilde{\Xi}_{i}\right\} \equiv \bigoplus_{\sigma= \pm}\left(\widetilde{\Xi}_{i} \otimes \widetilde{\Xi}_{i}\right)_{\sigma}, \\
\left(\widetilde{\Xi}_{i} \otimes \widetilde{\Xi}_{i}\right)_{ \pm} & =\bigoplus_{k=1}^{n_{\mathcal{C}}^{\mathbf{P}}}\left(\Xi_{k}\right)_{ \pm} \sum_{q=1}^{n_{\mathcal{C}}^{\tilde{\mathbf{P}}}} \frac{h_{q}}{g^{\widetilde{P}}} \chi_{\Xi_{k}}^{\mathbf{P}}\left(\mathcal{C}_{q}\right)^{*} \chi_{\left(\widetilde{\Xi}_{i} \otimes \widetilde{\Xi}_{i}\right)_{ \pm}}^{\widetilde{\mathbf{P}}}\left(\mathcal{C}_{q}\right), \\
\chi_{\left(\widetilde{\Xi}_{i} \otimes \widetilde{\Xi}_{i}\right)_{ \pm}}^{\widetilde{P}}(\widetilde{P}) & =\frac{1}{2}\left[\chi \underset{\widetilde{\Xi_{i}}}{\widetilde{P}}(\widetilde{P})^{2} \pm \chi_{\widetilde{\Xi}_{i}}^{\widetilde{\mathbf{P}}}\left(\widetilde{P}^{2}\right)\right] .
\end{aligned}
$$

Spinon-geminate-excitation-relevant direct-product representations of the double groups $\widetilde{\mathbf{I}}, \widetilde{\mathbf{T}}, \widetilde{\mathbf{O}}$, and $\widetilde{\mathbf{O}}_{\mathrm{h}}$ are listed with their containing single-valued irreducible representations of the corresponding point symmetry groups in Table I and with further details, including their characters, in Appendix C.

\section{RAMAN INTENSITY PROFILES}

\section{A. Point symmetry argument}

Within the LF theory [53-55], the Raman scattering intensity at absolute zero reads $[24,62]$

$$
\begin{aligned}
I(\omega)= & \frac{1}{2 \pi \hbar L} \int_{-\infty}^{\infty}\left\langle 0\left|e^{\frac{i \mathscr{H} t}{\hbar}} \mathscr{R} e^{-\frac{i \mathscr{H} t}{\hbar}} \mathscr{R}\right| 0\right\rangle e^{i \omega t} d t, \\
\mathscr{R} \equiv & -J \sum_{\lambda=x, y, z} \sum_{\langle m, n\rangle_{\lambda}}\left(\boldsymbol{e}_{\mathrm{in}} \cdot \boldsymbol{d}_{m n}\right)\left(\boldsymbol{e}_{\mathrm{sc}} \cdot \boldsymbol{d}_{m n}\right) \sigma_{m}^{\lambda} \sigma_{n}^{\lambda} \\
= & i J \sum_{\lambda=x, y, z} \sum_{\langle m, n\rangle_{\lambda}}\left(\boldsymbol{e}_{\mathrm{in}} \cdot \boldsymbol{d}_{m n}\right)\left(\boldsymbol{e}_{\mathrm{sc}} \cdot \boldsymbol{d}_{m n}\right) \\
& \times \hat{u}_{\langle m, n\rangle} c_{m} c_{n},
\end{aligned}
$$

where $\boldsymbol{e}_{\text {in }} \equiv\left(\sin \vartheta_{\text {in }} \cos \varphi_{\text {in }}, \sin \vartheta_{\text {in }} \sin \varphi_{\text {in }}, \cos \vartheta_{\text {in }}\right)$ and $\boldsymbol{e}_{\text {sc }} \equiv$ $\left(\sin \vartheta_{\mathrm{sc}} \cos \varphi_{\mathrm{sc}}, \sin \vartheta_{\mathrm{sc}} \sin \varphi_{\mathrm{sc}}, \cos \vartheta_{\mathrm{sc}}\right)$ are the polarization vectors of incident and scattered lights, respectively, while $\boldsymbol{d}_{m n} \equiv \boldsymbol{r}_{m}-\boldsymbol{r}_{n}$ are the lattice vectors, with $\boldsymbol{r}_{m}$ and $\boldsymbol{r}_{n}$ being the positions of neighboring sites. When the ground state belongs to the double group $\widetilde{\mathbf{P}}[63]$, it is useful to write the Raman operator (19) as [64]

$$
\mathscr{R}=\sum_{i}^{\prime} \sum_{\mu=1}^{d_{\mathbb{\Xi}_{i}}^{\widetilde{\mathbb{P}}}} E_{\widetilde{\Xi}_{i}: \mu}^{\widetilde{\mathbf{P}}} \mathcal{R}_{\widetilde{\Xi}_{i}: \mu}^{\widetilde{\widetilde{P}}}=\sum_{i}^{\prime} \sum_{\mu=1}^{d_{\Xi_{i}}^{\mathbf{P}}} E_{\Xi_{i}: \mu}^{\mathbf{P}} \mathcal{R}_{\Xi_{i}: \mu}^{\mathbf{P}},
$$

where $\sum_{i}^{\prime}$ runs over the LF-active irreducible representations $\widetilde{\Xi}_{i}$ of $\widetilde{\mathbf{P}}$, which are necessarily real and single-valued and therefore equal to the irreducible representations $\Xi_{i}$ of the corresponding point symmetry group $\mathbf{P}$, and $E_{\widetilde{\Xi}_{i: \mu}}^{\widetilde{\mathbb{P}}}\left(E_{\Xi_{i}: \mu}^{\mathbf{P}}\right)$ and $\mathcal{R}_{\widetilde{\Xi}_{i}: \mu}^{\widetilde{\widetilde{P}}}\left(\mathcal{R}_{\Xi_{i}: \mu}^{\mathbf{P}}\right)$ are the $\mu$ th polarization-vector basis function and LF vertex for $\widetilde{\Xi}_{i}\left(\Xi_{i}\right)$, respectively, both of which are explicitly given in Appendix D. Within the LF formulation, the nonvanishing vertices read $\mathcal{R}_{\mathrm{A}: \mu}^{\mathrm{I}}, \mathcal{R}_{\mathrm{H}: \mu}^{\mathrm{I}}$ for the dodecahedron, $\mathcal{R}_{\mathrm{A}: \mu}^{\mathrm{T}}, \mathcal{R}_{\mathrm{E}: \mu}^{\mathrm{T}}, \mathcal{R}_{\mathrm{T}: \mu}^{\mathrm{T}}$ for the truncated tetrahedron, $\mathcal{R}_{\mathrm{A}_{\mathrm{lg}}: \mu}^{\mathbf{O}_{\mathrm{h}}}$, $\mathcal{R}_{\mathrm{E}_{\mathrm{g}}: \mu}^{\mathbf{O}_{\mathrm{h}}}, \mathcal{R}_{\mathrm{T}_{2 \mathrm{~g}}: \mu}^{\mathbf{O}_{\mathrm{h}}}$ for the truncated octahedron, and, for reference, $\mathcal{R}_{\mathrm{A}_{1}: \mu}^{\mathrm{C}_{\mathrm{6v}}}, \mathcal{R}_{\mathrm{E}_{2}: \mu}^{\mathrm{C}_{\mathrm{Gv}}}$ for two-dimensional lattices of triangular geometry [62,65-67]. In the spherical lattice geometry realized by Platonic and Archimedean polyhedra, all the vertices of the identity representation, such as $\mathcal{R}_{\mathrm{A}: \mu}^{\mathrm{I}}, \mathcal{R}_{\mathrm{A}: \mu}^{\mathrm{T}}$, and $\mathcal{R}_{\mathrm{A}_{\mathrm{h} g}: \mu}^{\mathbf{O}_{\mathrm{g}}}$, commute with the corresponding Hamiltonians and therefore

\begin{tabular}{|c|c|c|}
\hline$\widetilde{\mathbf{P}}$ & $\widetilde{\Xi}_{i} \otimes \widetilde{\Xi}_{j}$ & $\bigoplus_{k} \widetilde{\Xi}_{k}=\bigoplus_{k} \Xi_{k}$ \\
\hline$\widetilde{\mathbf{I}}$ & $\begin{array}{c}\left\{\mathrm{I}_{\frac{5}{2}} \otimes \mathrm{I}_{\frac{5}{2}}\right\} \\
\mathrm{I}_{\frac{5}{2}} \otimes \mathrm{G}_{\frac{3}{2}} \\
\left\{\mathrm{G}_{\frac{3}{2}} \otimes \mathrm{G}_{\frac{3}{2}}\right\}\end{array}$ & $\begin{array}{l}\{\underline{\mathrm{A}}\} \oplus\{\mathrm{G}\} \oplus 2\{\underline{\underline{H}}\} \\
\mathrm{T}_{1} \oplus \mathrm{T}_{2} \oplus 2 \mathrm{G} \oplus 2 \underline{\underline{\mathrm{H}}} \\
\{\underline{\mathrm{A}}\} \oplus\{\underline{\underline{\mathrm{H}}}\}\end{array}$ \\
\hline$\widetilde{\mathbf{T}}$ & $\begin{aligned}\left\{G_{\frac{3}{2}}^{(2)}\right. & \left.\otimes G_{\frac{3}{2}}^{2}\right\} \\
G_{\frac{3}{2}}^{(2)} & \otimes E_{\frac{1}{2}}^{(2)} \\
\left\{E_{\frac{1}{2}}\right. & \left.\otimes E_{\frac{1}{2}}\right\} \\
G_{\frac{3}{(1)}}^{(1)} & \otimes G_{\frac{3}{2}}^{(2)} \\
G_{\frac{3}{2}}^{(1)} & \otimes E_{\frac{1}{2}} \\
\left\{G_{\frac{3}{2}}^{(2)}\right. & \left.\otimes G_{\frac{3}{2}}^{(1)}\right\}\end{aligned}$ & $\begin{array}{l}\underline{\underline{\left.\mathrm{E}^{(1)}\right\}}} \\
\underline{\underline{\mathrm{E}^{(2)}}} \oplus \underline{\underline{\mathrm{T}}} \\
\underline{\underline{\mathrm{A}}} \oplus \underline{\underline{T}} \\
\underline{\underline{\mathrm{E}^{(1)}}} \oplus \underline{\underline{T}} \\
\left.\underline{\underline{\underline{\mathrm{E}^{(2)}}}}\right\}\end{array}$ \\
\hline$\widetilde{\mathbf{O}}$ & $\begin{aligned}\left\{\mathrm{E}_{\frac{1}{2}}\right. & \left.\otimes \mathrm{E}_{\frac{1}{2}}\right\} \\
\mathrm{E}_{\frac{1}{2}} & \otimes \mathrm{E}_{\frac{5}{2}} \\
\left\{\mathrm{E}_{\frac{5}{2}}\right. & \left.\otimes \mathrm{E}_{\frac{5}{2}}\right\} \\
\mathrm{G}_{\frac{3}{2}} & \otimes \mathrm{E}_{\frac{1}{2}} \\
\mathrm{G}_{\frac{3}{2}} & \otimes \mathrm{E}_{\frac{5}{2}} \\
\mathrm{G}_{\frac{3}{2}} & \otimes \mathrm{G}_{\frac{3}{2}}\end{aligned}$ & 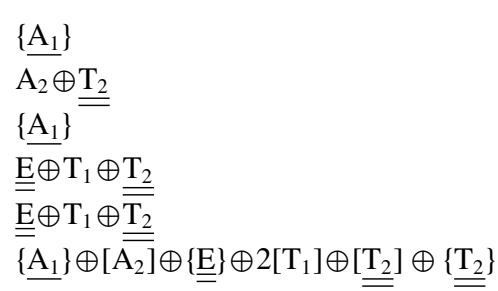 \\
\hline$\widetilde{\mathrm{O}_{\mathrm{h}}}$ & $\begin{array}{c}\left\{\mathrm{G}_{\frac{1}{2}+\frac{5}{2}} \otimes \mathrm{G}_{\frac{1}{2}+\frac{5}{2}}\right\} \\
\mathrm{G}_{\frac{3}{2}}^{\mathrm{g}} \otimes \mathrm{G}_{\frac{1}{2}+\frac{5}{2}} \\
\left\{\mathrm{G}_{\frac{3}{3}}^{\mathrm{g}} \otimes \mathrm{G}_{\frac{3}{2}}^{\mathrm{g}}\right\} \\
\mathrm{G}_{\frac{3}{2}}^{\mathrm{u}} \otimes \mathrm{G}_{\frac{1}{2}+\frac{5}{2}} \\
\mathrm{G}_{\frac{3}{2}}^{\mathrm{g}} \otimes \mathrm{G}_{\frac{3}{2}}^{\mathrm{u}} \\
\left\{\mathrm{G}_{\frac{3}{2}}^{\mathrm{u}} \otimes \mathrm{G}_{\frac{3}{2}}^{\mathrm{u}}\right\}\end{array}$ & 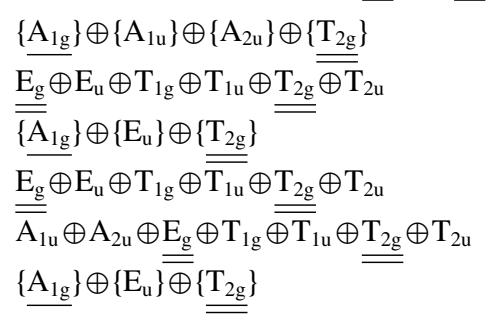 \\
\hline
\end{tabular}

TABLE I. Spinon-geminate-excitation-relevant direct-product representations composed of double-valued irreducible representations $\widetilde{\Xi}_{i} \otimes \widetilde{\Xi}_{j}$ and their decompositions into single-valued irreducible representations $\widetilde{\Xi}_{k}$, which are doubly or singly underlined when they are relevant to inelastic (Raman) or elastic (Rayleigh) scatterings, respectively, for various double groups $\widetilde{\mathbf{P}}$. Note that $\widetilde{\Xi}_{k}$ of $\widetilde{\mathbf{P}}$ is nothing but $\Xi_{k}$ of $\mathbf{P}$.

reduce to Rayleigh scattering. This is the case for $\mathcal{R}_{\mathrm{A}_{1}: \mu}^{\mathrm{C}_{\mathrm{v}}}$ as well.

Since the ground state (9) is invariant under every symmetry operation of $\mathbf{P}$, every expectation value between Raman vertices of different symmetry species for it goes to 0 $[62,64,66]$,

$$
\begin{aligned}
& \frac{1}{2 \pi \hbar L} \int_{-\infty}^{\infty}\left\langle 0\left|e^{\frac{i \mathscr{H} t}{\hbar}} \mathcal{R}_{\Xi_{i}: \mu}^{\mathbf{P}} e^{-\frac{i \mathscr{H} t}{\hbar}} \mathcal{R}_{\Xi_{j}: \nu}^{\mathbf{P}}\right| 0\right\rangle e^{i \omega t} d t \\
& \quad=\frac{\delta_{i j} \delta_{\mu v}}{2 \pi \hbar L} \int_{-\infty}^{\infty}\left\langle 0\left|e^{\frac{i \mathscr{H} t}{\hbar}} \mathcal{R}_{\Xi_{i}: \mu}^{\mathbf{P}} e^{-\frac{i \mathscr{H} t}{\hbar}} \mathcal{R}_{\Xi_{i}: \mu}^{\mathbf{P}}\right| 0\right\rangle e^{i \omega t} d t \\
& \quad \equiv \delta_{i j} \delta_{\mu \nu} I_{\Xi_{i}: \mu}^{\mathbf{P}}(\omega),
\end{aligned}
$$

and $I_{\Xi_{i}: \mu}^{\mathbf{P}}(\omega) \quad\left(\mu=1, \ldots, d_{\Xi_{i}}^{\mathbf{P}}\right)$ no longer depend on $\mu$ $[24,29,62,65]$. While the Raman spectra of gauge-ground Kitaev polyhedra are analyzable with direct-product representations of their projective symmetry groups $\widetilde{\mathbf{P}}$, they can be classified by irreducible representations of the corresponding point symmetry groups $\mathbf{P}$. Substituting the irreducible decomposition of the Raman operator $\mathscr{R}$ (20) into the LF expression of the Raman intensity (19) and taking account of the spectral degeneracy within each multidimensional irreducible 

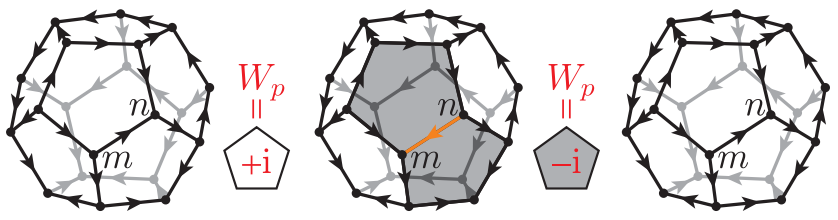

(a) $\left|\left\{W_{p}\right\}\right\rangle_{0}$ (c) $\sigma_{m}^{z} \sigma_{n}^{z}\left|\left\{W_{p}\right\}\right\rangle_{0}$

FIG. 3. Actions of the spin operators $\sigma_{n}^{z}=i c_{n} \eta_{n}^{z}$ (b) and $\sigma_{m}^{z} \sigma_{n}^{z}=$ $-i \hat{u}_{\langle m, n\rangle_{z}} c_{m} c_{n}$ (c) on the gauge-ground Kitaev dodecahedron $\left|\left\{W_{p}\right\}\right\rangle_{0}$ (a) in the context of calculating the dynamic structure factor (24) and Raman scattering intensity (23).

representation (cf. Appendix D), we have

$$
\begin{aligned}
I(\omega) & =\sum_{i}^{\prime} \sum_{\mu=1}^{d_{\Xi_{i}}^{\mathbf{P}}}\left(E_{\Xi_{i}: \mu}^{\mathbf{P}}\right)^{2} I_{\Xi_{i}: \mu}^{\mathbf{P}}(\omega) \\
& =\sum_{i}^{\prime} I_{\Xi_{i}: 1}^{\mathbf{P}}(\omega) \sum_{\mu=1}^{d_{\Xi_{i}}^{\mathbf{P}}}\left(E_{\Xi_{i}: \mu}^{\mathbf{P}}\right)^{2} .
\end{aligned}
$$

Bearing in mind that $\left[\mathcal{R}_{\Xi_{i}: \mu}^{\mathbf{P}}, \hat{W}_{p}\right]=0,{ }_{q}\left\langle\left\{W_{p}\right\} \mid\left\{W_{p}\right\}\right\rangle_{q^{\prime}}=\delta_{q q^{\prime}}$, and $c_{l}|0\rangle=\sum_{k=1}^{L / 2}\left(\psi_{l, 2 k-1}+i \psi_{l, 2 k}\right) \alpha_{k}^{\dagger}|0\rangle$, the LF vertex $\mathcal{R}_{\Xi_{i:}: \mu}^{\mathbf{P}}$ evokes two spinons without any vison (for more details refer to Appendix D),

$$
\begin{aligned}
I_{\Xi_{i}: \mu}^{\mathbf{P}}(\omega)= & \int_{-\infty}^{\infty} \frac{d t e^{i \omega t}}{2 \pi \hbar L} \sum_{q=0}^{2^{\frac{L}{2}+1}-1} \sum_{\kappa=0}^{\frac{L}{2}-1} 0\left\langle\left\{W_{p}\right\}\right| \otimes_{0}\left\langle\left\{n_{k}\right\}\right| \\
& \times e^{\frac{i \mathscr{H} t}{\hbar}} \mathcal{R}_{\Xi_{i}: \mu}^{\mathbf{P}} e^{-\frac{i \mathscr{H} t}{\hbar}}\left|\left\{n_{k}\right\}\right\rangle_{\kappa} \otimes\left|\left\{W_{p}\right\}\right\rangle_{q} \\
& \times{ }_{q}\left\langle\left\{W_{p}\right\}\left|\otimes_{\kappa}\left\langle\left\{n_{k}\right\}\left|\mathcal{R}_{\Xi_{i: \mu}: \mu}^{\mathbf{P}}\right|\left\{n_{k}\right\}\right\rangle_{0} \otimes\right|\left\{W_{p}\right\}\right\rangle_{0} \\
= & \frac{1}{L} \sum_{1=k<k^{\prime}=\frac{L}{2}}\left|\left\langle 0\left|\alpha_{k} \alpha_{k^{\prime}} \mathcal{R}_{\Xi_{i}: \mu}^{\mathbf{P}}\right| 0\right\rangle\right|^{2} \\
& \times \delta\left(\hbar \omega-\varepsilon_{k}-\varepsilon_{k^{\prime}}\right) .
\end{aligned}
$$

We may be reminded that the above is not the case with any single spin operator. Unlike the Raman response, visons (Fig. 3) as well as spinons are involved in the dynamic spin response $[68,69]$ :

$$
\begin{aligned}
& S^{\lambda \lambda}(\boldsymbol{q}, \omega)=\frac{1}{2 \pi \hbar L} \int_{-\infty}^{\infty} \sum_{m, n=1}^{L} e^{-i \boldsymbol{q} \cdot\left(\boldsymbol{r}_{m}-\boldsymbol{r}_{n}\right)} \\
& \times\left\langle 0\left|e^{\frac{i \mathscr{H} e_{t}}{\hbar}} \sigma_{m}^{\lambda} e^{-\frac{i \mathscr{H} t}{\hbar}} \sigma_{n}^{\lambda}\right| 0\right\rangle e^{i \omega t} d t \\
& =\int_{-\infty}^{\infty} \frac{d t e^{i \omega t}}{2 \pi \hbar L} \sum_{m, n=1}^{L} \sum_{q=0}^{2 \frac{L}{2}+1} \sum_{\kappa=0}^{2^{\frac{L}{2}-1}-1} e^{-i \boldsymbol{q} \cdot\left(\boldsymbol{r}_{m}-\boldsymbol{r}_{n}\right)} \\
& \times{ }_{0}\left\langle\left\{W_{p}\right\}\right| \otimes_{0}\left\langle\left\{n_{k}\right\}\left|e^{\frac{i \mathscr{H} t_{t}}{\hbar}} \sigma_{m}^{\lambda} e^{-\frac{i \mathscr{H} t_{t}}{\hbar}}\right|\left\{n_{k}^{\prime}\right\}\right\rangle_{\kappa} \\
& \otimes\left|\left\{W_{p}\right\}\right\rangle_{q q}\left\langle\left\{W_{p}\right\}\left|\otimes{ }_{\kappa}\left\langle\left\{n_{k}^{\prime}\right\}\left|\sigma_{n}^{\lambda}\right|\left\{n_{k}\right\}\right\rangle_{0} \otimes\right|\left\{W_{p}\right\}\right\rangle_{0} .
\end{aligned}
$$

Indeed ${ }_{0}\left\langle\left\{n_{k}\right\}\left|\alpha_{k^{\prime}}^{\dagger} \alpha_{k^{\prime}}\right|\left\{n_{k}\right\}\right\rangle_{0}=0 \quad\left(k^{\prime}=1, \ldots, \frac{L}{2}\right)$, but the spinon operator $\alpha_{k}$ and therefore the vacuum state $\left|\left\{n_{k}\right\}\right\rangle_{0}$ depend on the background flux configuration $\left|\left\{W_{p}\right\}\right\rangle_{q}$. We denote these against the excited flux configuration $\left|\left\{W_{p}\right\}\right\rangle_{q \neq 0}$ by $\alpha_{k}^{\prime}$ and $\left|\left\{n_{k}^{\prime}\right\}\right\rangle_{0}$ distinguishably from $\alpha_{k}$ and $\left|\left\{n_{k}\right\}\right\rangle_{0}$ against
$\left|\left\{W_{p}\right\}\right\rangle_{0}$ in Eq. (24). Since spinons in an excited flux sector read a linear combination of spinons in the ground flux sector, $\alpha_{k^{\prime}}^{\prime}=\sum_{k=1}^{L / 2}\left(\chi_{k^{\prime}, k} \alpha_{k}+v_{k^{\prime}, k} \alpha_{k}^{\dagger}\right)\left(k^{\prime}=1, \ldots, \frac{L}{2}\right)$, and their vacuum $\left|\left\{n_{k}^{\prime}\right\}\right\rangle_{0}$ reads a linear combination of the groundflux-sector spinon vacuum and/or excited states, i.e., a linear combination of $\left|\left\{n_{k}\right\}\right\rangle_{0}, \alpha_{k_{1}}^{\dagger} \alpha_{k_{2}}^{\dagger}\left|\left\{n_{k}\right\}\right\rangle_{0}, \alpha_{k_{1}}^{\dagger} \alpha_{k_{2}}^{\dagger} \alpha_{k_{3}}^{\dagger} \alpha_{k_{4}}^{\dagger}\left|\left\{n_{k}\right\}\right\rangle_{0}, \ldots$ or a linear combination of $\alpha_{k_{1}}^{\dagger}\left|\left\{n_{k}\right\}\right\rangle_{0}, \alpha_{k_{1}}^{\dagger} \alpha_{k_{2}}^{\dagger} \alpha_{k_{3}}^{\dagger}\left|\left\{n_{k}\right\}\right\rangle_{0}, \ldots$, we can exactly calculate the dynamic structure factor (24) as well [69]. In higher dimensions, Eq. (24) is hard to calculate for sufficiently large systems, with excited flux configurations $\left|\left\{W_{p}\right\}\right\rangle_{q \neq 0}$ no longer being invariant under the primitive translation, but we can employ a Dyson equation instead to accomplish the thermodynamic-limit calculation $[68,69]$.

Figure 2 shows the polarized Raman spectra of gaugeground Kitaev spin balls with light polarization vectors varying within the $x y$ plane. The polarization dependence of the intensity is very weak in the dodecahedron but significant and individual in the truncated tetrahedron and octahedron. The former observations are similar to the case for the honeycomb Kitaev QSL [24]. For polarization vectors in the $x y$ plane, $\vartheta_{\text {in }}=\vartheta_{\mathrm{sc}}=\frac{\pi}{2}$ with varying $\varphi_{\text {in }}$ and $\varphi_{\mathrm{sc}}$, we have

$$
\begin{aligned}
\sum_{\mu=1}^{2}\left(E_{\mathrm{E}_{2}: \mu}^{\mathbf{C}_{\mathrm{v}}}\right)^{2} & =\frac{1}{2} \\
\sum_{\mu=1}^{5}\left(E_{\mathrm{H}: \mu}^{\mathbf{I}}\right)^{2} & =\frac{\cos ^{2}\left(\varphi_{\mathrm{in}}-\varphi_{\mathrm{sc}}\right)}{6}+\frac{1}{2}, \\
\sum_{\mu=1}^{2}\left(E_{\mathrm{E}: \mu}^{\mathbf{T}}\right)^{2} & =\sum_{\mu=1}^{2}\left(E_{\mathrm{E}_{\mathrm{g}}: \mu}^{\mathbf{O}_{\mathrm{h}}}\right)^{2} \\
& =\frac{\cos ^{2}\left(\varphi_{\mathrm{in}}-\varphi_{\mathrm{sc}}\right)}{6}+\frac{\cos ^{2}\left(\varphi_{\mathrm{in}}+\varphi_{\mathrm{sc}}\right)}{2}, \\
\sum_{\mu=1}^{3}\left(E_{\mathrm{T}: \mu}^{\mathbf{T}}\right)^{2} & =\sum_{\mu=1}^{3}\left(E_{\mathrm{T}_{2 \mathrm{~g}}: \mu}^{\mathbf{O}_{\mathrm{h}}}\right)^{2}=\frac{\sin ^{2}\left(\varphi_{\mathrm{in}}+\varphi_{\mathrm{sc}}\right)}{2} ;
\end{aligned}
$$

hence the perfect depolarization of Raman scattering in a honeycomb QSL. While the $\widetilde{\mathbf{I}}$ gauged dodecahedron also has one and only a Raman-active multidimensional irreducible representation and all three relevant direct-product representations of $\widetilde{\mathbf{I}}$ contain this $\mathrm{H}$ mode, the sum of its five basis functions no longer reduces to a constant, resulting in similar shapes peaked at the three fixed frequencies $\hbar \omega / 2 J=2,1+$ $\sqrt{6}$, and $2 \sqrt{6}$ but different weights varying as Eq. (26) of the polarized spectra. The $\widetilde{\mathbf{T}}$ and $\widetilde{\mathbf{O}_{\mathrm{h}}}$ gauged polyhedra each have two Raman-active modes to yield spectra peaking and weighing differently according to the light polarization. Such observations are also the case for $\widetilde{\mathbf{D}_{2 \mathrm{~h}}}$ harmonic honeycomb Kitaev QSLs in three dimensions [42,62]. Full details of the polarized Raman intensity profiles of all the gauged polyhedra in question are given in Appendix D.

\section{B. Projective symmetry argument}

The $\widetilde{\mathbf{T}}$ and $\widetilde{\mathbf{O}_{\mathrm{h}}}$ gauged polyhedra each have three spinon modes to yield geminate excitations of $3+{ }_{3} \mathrm{C}_{2}$ types. There are six pair-spinon-resonant frequencies in each of them. In the case of $\widetilde{\mathbf{T}}$, one of them, $\left\{\mathrm{E}_{\frac{1}{2}} \otimes \mathrm{E}_{\frac{1}{2}}\right\}(\hbar \omega / 2 J=2 \sqrt{2})$, is a Rayleigh channel, while all the rest contain the Ramanactive $\mathrm{E}$ (detectable with $\varphi_{\text {in }} \pm \varphi_{\mathrm{sc}} \neq \frac{\pi}{2}$ ) and/or T (detectable 
with $\left.\varphi_{\text {in }}+\varphi_{\mathrm{sc}} \neq 0, \pi\right)$ modes where the two-dimensional real irreducible representation $\mathrm{E} \equiv \mathrm{E}^{(1)} \oplus \mathrm{E}^{(2)}$ splits into two onedimensional complex ones, $\mathrm{E}^{(1)}$ and $\mathrm{E}^{(2)}$, causing nonvanishing Raman intensities at all six frequencies but $\hbar \omega / 2 J=$ $2 \sqrt{2}$. In the case of $\widetilde{\mathbf{O}_{\mathrm{h}}}$, all the direct-product representations contain the Raman-active $\mathrm{T}_{2 \mathrm{~g}}$ mode (detectable with $\varphi_{\text {in }}+$ $\left.\varphi_{\mathrm{sc}} \neq 0, \pi\right)$, causing nonvanishing Raman intensities at all six frequencies. On the other hand, only the three direct-product representations $\mathrm{G}_{\frac{3}{2}}^{\mathrm{g}} \otimes \mathrm{G}_{\frac{1}{2}+\frac{5}{2}}(\hbar \omega / 2 J=1+\sqrt{4-\sqrt{3}}), \mathrm{G}_{\frac{3}{2}}^{\mathrm{u}} \otimes$ $\mathrm{G}_{\frac{1}{2}+\frac{5}{2}}(\hbar \omega / 2 J=1+\sqrt{4+\sqrt{3}})$, and $\mathrm{G}_{\frac{3}{2}}^{\mathrm{g}} \otimes \mathrm{G}_{\frac{3}{2}}^{\mathrm{u}}(\hbar \omega / 2 J=$ $\sqrt{4-\sqrt{3}}+\sqrt{4+\sqrt{3}})$ contain another Raman-active mode, $\mathrm{E}_{\mathrm{g}}$ (detectable with $\varphi_{\text {in }} \pm \varphi_{\mathrm{sc}} \neq \frac{\pi}{2}$ ). In this context, we should pay special attention to the geminate excitations, labeled $\left\{\mathrm{G}_{\frac{3}{2}}^{\mathrm{g}} \otimes \mathrm{G}_{\frac{3}{2}}^{\mathrm{g}}\right\}(\hbar \omega / 2 J=2 \sqrt{4-\sqrt{3}})$ and $\left\{\mathrm{G}_{\frac{3}{2}}^{\mathrm{u}} \otimes \mathrm{G}_{\frac{3}{2}}^{\mathrm{u}}\right\}(\hbar \omega / 2 J=$ $\left.2 \sqrt{\frac{\sqrt{2}}{4}+\sqrt{3}}\right)$. If we describe this gauged polyhedron in terms of $\widetilde{\mathbf{O}}$, rather than $\widetilde{\mathbf{O}_{\mathrm{h}}}$, these two direct-product representations degenerate into $\left\{\mathrm{G}_{\frac{3}{2}} \otimes \mathrm{G}_{\frac{3}{2}}\right\}=\left\{\underline{\mathrm{A}_{1}}\right\} \oplus\{\underline{\underline{E}}\} \oplus\left\{\underline{\underline{T_{2}}}\right\}$ (see Table I), leading to misunderstanding as if outgoing photons of $\varphi_{\mathrm{sc}}=\varphi_{\text {in }}$ cause nonvanishing Raman intensities at the two frequencies $\hbar \omega / 2 J=2 \sqrt{4 \mp \sqrt{3}}$ as well. Under the pertinent $\widetilde{\mathbf{O}_{\mathrm{h}}}$ description, the Raman intensities at the two frequencies $\hbar \omega / 2 J=2 \sqrt{4 \mp \sqrt{3}}$ in the gauged truncated octahedron belong definitely to the $\mathrm{T}_{2 \mathrm{~g}}$ symmetry species, because they are mediated by spinon geminate excitations belonging to the direct-product representations $\left\{\mathrm{G}_{\frac{3}{2}}^{\mathrm{g}} \otimes \mathrm{G}_{\frac{3}{2}}^{\mathrm{g}}\right\}$ and $\left\{\mathrm{G}_{\frac{3}{2}}^{\mathrm{u}} \otimes \mathrm{G}_{\frac{3}{2}}^{\mathrm{u}}\right\}$, both of which decompose into $\left\{\underline{\mathrm{A}_{1 \mathrm{~g}}}\right\} \oplus^{\frac{3}{2}}\left\{\mathrm{E}_{\mathrm{u}}\right\} \oplus\left\{\underline{\mathrm{T}_{2 \mathrm{~g}}}\right\}$, i.e., the Raman-active $\mathrm{T}_{2 \mathrm{~g}}$, LF-Raman-inactive $\mathrm{A}_{1 \mathrm{~g}}$, and $\overline{\overline{\mathrm{d}}}$ amaninactive $\mathrm{E}_{\mathrm{u}}$ (instead of Raman-active $\mathrm{E}_{\mathrm{g}}$ ) symmetry species (see Table I).

In an attempt to describe partons in the Kitaev truncated octahedron, MPT [49] restrict their symmetry argument to gauged rotations $\widetilde{\mathbf{R}} \subset \mathrm{SU}(2) \cong \operatorname{Spin}(3)$, i.e., double covers of pure rotation groups $\mathbf{R} \subset \mathrm{SO}(3)$, because they employ projective symmetry groups with the aim of characterizing an itinerant parton as a charged particle in quantized orbital motion and, therefore, require the isomorphism $\mathrm{SU}(2) / \mathbb{Z}_{2} \cong \mathrm{SO}(3)$. For partons emergent in a gauged truncated octahedron, they consider gauging the subgroup $\mathbf{O}$ of the full octahedral group $\mathbf{O}_{\mathrm{h}}$. On the other hand, in order to describe spinon geminate, rather than single, excitations in the context of Raman scattering, we construct and have to construct the double cover of $\mathbf{O}_{\mathrm{h}} \subset \mathrm{O}(3)$ [70] instead of that of $\mathbf{O} \subset \mathrm{SO}(3)$. Not until we analyze the projective symmetry of Majorana spinons to the fullest extent can we correctly understand Raman scattering in a time-reversal-invariant gauged polyhedron.

\section{SUMMARY AND FUTURE ASPECTS}

Our approach to Raman observations of QSLs is feasible regardless of the geometry. Kitaev nanoribbons [45,46], for instance, are describable with gauged space groups, $\mathbf{L} \wedge \widetilde{\mathbf{P}}$, where $\mathbf{L}$ is a one-dimensional translation group [71]. Their eigenspectra are no longer discrete but consist of continuous bands. Intraband and interband spinon geminate excitations are distinguished and identified by $\underset{\sim}{\mathbb{P}}$ light polarizations and direct-product representations of $\mathbf{L} \wedge \widetilde{\mathbf{P}}$ [72].
Another extension of our approach is going beyond the LF vertices [26,27]. In the $\widetilde{\mathbf{T}}$ Kitaev spin ball, the direct-product representation $\left\{E_{\frac{1}{2}} \otimes E_{\frac{1}{2}}\right\}$ is Raman inactive within the LF scheme (Table I), but an $\mathrm{E}_{\frac{1}{2}}$ multiple direct-product representation may become Raman active in higher-order scatterings to visualize the Majorana spinon spectrum in a wider range. Optical observation of partons in QSLs will be even more attractive with the language of projective symmetry.

\section{ACKNOWLEDGMENT}

This work was supported by the Ministry of Education, Culture, Sports, Science, and Technology of Japan.

\section{APPENDIX A: PROJECTIVE SYMMETRY OPERATIONS ON GAUGE-GROUND KITAEV POLYHEDRA}

Dodecahedral, truncated-tetrahedral, and truncatedoctahedral lattices belong to the point symmetry groups $\mathbf{I}_{\mathrm{h}}, \mathbf{T}_{\mathrm{d}}$, and $\mathbf{O}_{\mathrm{h}}$, respectively. We illustrate their symmetry operations in Fig. 4. When we consider Kitaev models of these lattices, their free Majorana fermionic Hamiltonians with given gauge fields are not generally invariant under the point group actions of their belonging lattices. Let us find gauged point symmetry operations of the ground gauge sectors of these Hamiltonians. We illustrate symmetry operations of gauge-ground polyhedra in Fig. 5. Every gauge-ground Kitaev spin ball is such that all $W_{p}$ 's of $N_{p}=0$ $\bmod 4$ are -1 , all $W_{p}$ 's of $N_{p}=2 \bmod 4$ are +1 , and all $W_{p}$ 's of odd $N_{p}$ are either of $+i$ and $-i$ [49]. Since the Kitaev spin Hamiltonian is time reversal invariant, its ground state is at least doubly degenerate unless all $N_{p}$ 's are even [52].

Figure 5(a) illustrates a gauged rotation of the gauge-ground Kitaev dodecahedron. Suppose we rotate it by $\frac{2 \pi}{3}$ about one of the threefold axes $\boldsymbol{n}$, which we denote by $R\left(\frac{2 \pi}{3}, \boldsymbol{n}\right)$, and then gauge some Majorana fermions as $c_{l} \rightarrow-c_{l}$ or, equivalently, change the signs of their relevant bonds as $u_{\left\langle l, l^{\prime}\right\rangle_{\lambda}} \rightarrow-u_{\left\langle l, l^{\prime}\right\rangle_{\lambda}}(\lambda=x, y, z)$, so as to recover the initial bond configuration. When the rotational symmetry operation $R(\varphi, \boldsymbol{n})(0 \leqslant \varphi<2 \pi)$ is performed, there exist two such local gauge operations, which we denote by $\pm \Lambda[R(\varphi, \boldsymbol{n})]$, remembering the double-valued nature of rotation operators acting on half-integral spin states. In the example in Fig. $5(\mathrm{a}),-\Lambda\left[R\left(\frac{2 \pi}{3}, \boldsymbol{n}\right)\right]$ acts on two sites, while $+\Lambda\left[R\left(\frac{2 \pi}{3}, \boldsymbol{n}\right)\right]$ acts on all the rest, where we make site assignment to $\pm \Lambda[R(\varphi, \boldsymbol{n})]$ in accordance with SU(2) rotations. How many and which sites to operate depend not only on the rotation axis $\boldsymbol{n}$ and angle $\varphi$ but also on the initial bond configuration. We have $2^{\frac{L}{2}+1}$ flux configurations $\left\{W_{p}\right\}$ including the ground two, each available from a set of $2^{\frac{3 L}{2}} / 2^{\frac{L}{2}+1}=2^{L-1}$ different bond configurations $\left\{u_{\langle m, n\rangle_{\lambda}}\right\}$. We denote a couple of these serial transformations as $+\Lambda\left[R\left(\frac{2 \pi}{3}, \boldsymbol{n}\right)\right] R\left(\frac{2 \pi}{3}, \boldsymbol{n}\right) \equiv$ $\overline{R\left(\frac{2 \pi}{3}, \boldsymbol{n}\right)} \quad$ and $\quad-\Lambda\left[R\left(\frac{2 \pi}{3}, \boldsymbol{n}\right)\right] R\left(\frac{2 \pi}{3}, \boldsymbol{n}\right) \equiv R\left(\frac{2 \pi}{3}, \boldsymbol{n}\right)$. Note that $\left[\overline{R\left(\frac{2 \pi}{3}, \boldsymbol{n}\right)}\right]^{3}\left\{u_{\langle m, n\rangle_{\lambda}}\right\}=-\left\{u_{\langle m, n\rangle_{\lambda}}\right\}$, while $\left[R\left(\frac{2 \pi}{3}, \boldsymbol{n}\right)\right]^{3}\left\{u_{\langle m, n\rangle_{\lambda}}\right\}=\left\{u_{\langle m, n\rangle_{\lambda}}\right\}$.

Figure 5(a) illustrates inversion of the gauge-ground Kitaev dodecahedron as well, resulting in all $W_{p}$ 's being 


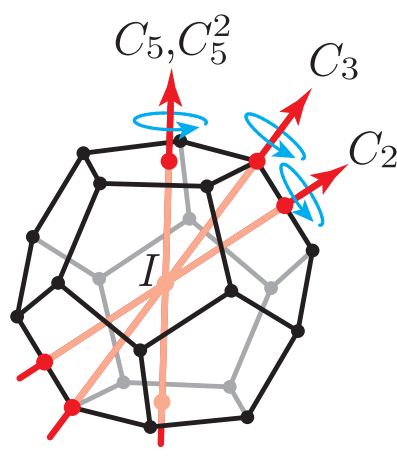

(a) $\mathbf{I}_{\mathrm{h}}$

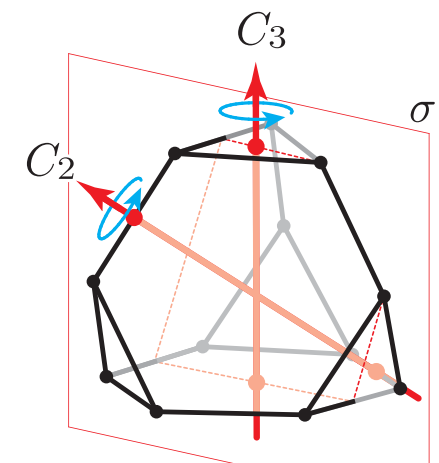

(b) $\mathbf{T}_{\mathrm{d}}$

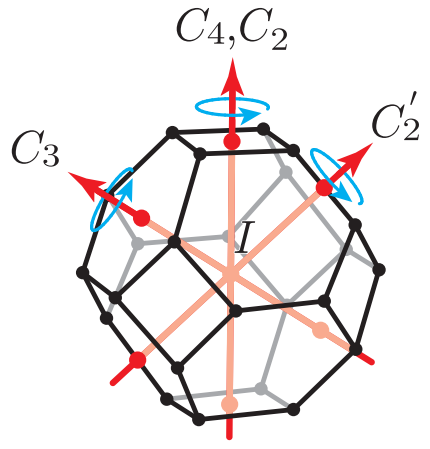

(c) $\mathrm{O}_{\mathrm{h}}$

FIG. 4. Point symmetry operations on (a) dodecahedral, (b) truncated-tetrahedral, and (c) truncated-octahedral lattices belonging to the full icosahedral $\left(\mathbf{I}_{\mathrm{h}}\right)$, tetrahedral $\left(\mathbf{T}_{\mathrm{d}}\right)$, and octahedral $\left(\mathbf{O}_{\mathrm{h}}\right)$ groups, respectively.

reversed, $\quad\left\{W_{p}=+i ; p=1, \ldots, 12\right\} \rightarrow\left\{W_{p}=-i ; p=\right.$ $1, \ldots, 12\}$. The constituent pentagons each initially have a flux of $\frac{\pi}{2}$ and all their fluxes $\Phi_{p}$ are reversed into $-\Phi_{p}$ by inversion. The flux variables $W_{p} \equiv e^{i \Phi_{p}}$ are also all reversed. Any local gauge transformation $c_{l} \rightarrow-c_{l}$ results in reversing the signs of bonds in pairs in the three surrounding polygons and therefore causes no change in their $W_{p}$ 's. We find that the symmetry group of the gauge-ground Kitaev dodecahedron is not the double cover of the full point symmetry group, $\widetilde{\mathbf{I}_{h}}$, but that of an $\mathrm{SO}(3)$ subgroup, $\widetilde{\mathbf{I}}$. This is the case with the gauge-ground Kitaev truncated tetrahedron as well [Fig. 5(b)]. Since the mirror operation $\sigma \in \mathbf{T}_{\mathrm{d}}$ reverses the $W_{p}$ 's of its four constituent triangles, its symmetry group is not $\widetilde{\mathbf{T}_{\mathrm{d}}}$ but $\widetilde{\mathbf{T}}$. On the other hand, inversion causes no change in $W_{p}$ 's of the gauge-ground truncated octahedron [Fig. 5(c)]. This is because the truncated octahedron consists of only $2 l$-sided polygons $(l \in \mathbb{N})$, whose fluxes are either 0 or $\pi$. Even though inversion reverses such fluxes as $\Phi_{p} \rightarrow-\Phi_{p}$, the corresponding flux variables $W_{p} \equiv e^{i \Phi_{p}}$ remain unchanged. Any two bond configurations $\left\{u_{\langle m, n\rangle_{\lambda}}\right\}$ yielding the same set of fluxes $\left\{W_{p}\right\}$ can be converted to each other by local gauge operations. Inversion of the gauge-ground truncated octahedron is also followed by two local gauge operations so as to recover the initial bond configuration, which we denote by $\pm \Lambda(I)$, each to act on different halves of the lattice sites. We generally denote a couple of gauged point symmetry operations $\pm \Lambda(P) P$ unifiedly as $\widetilde{P}$ and distinguishably by $\bar{P}$ and $\underline{P}$.

\section{APPENDIX B: IRREDUCIBLE REPRESENTATIONS OF DOUBLE GROUPS FOR GAUGE-GROUND KITAEV POLYHEDRA}

We denote the orders of a point symmetry group $\mathbf{P}$ and its double covering group $\widetilde{\mathbf{P}}$ by $g^{\mathbf{P}}$ and $g^{\widetilde{\mathbf{P}}}$, respectively. Suppose the double cover $\widetilde{\mathbf{P}}$ is the $\mathbb{Z}_{2}$-gauge extension of $\mathbf{P} \subset \mathrm{O}(3)$. Two group elements, $\widetilde{P}_{1} \in \widetilde{\mathbf{P}}$ and $\widetilde{P}_{2} \in \widetilde{\mathbf{P}}$, are conjugate when we find such an element $\widetilde{P} \in \widetilde{\mathbf{P}}$ as to satisfy

$$
\widetilde{P}_{2}=\widetilde{P} \widetilde{P}_{1} \widetilde{P}^{-1} \text {. }
$$

Every set of conjugate elements forms a class. The classes of the double groups of our interest read as follows.

$$
\begin{aligned}
\widetilde{\mathbf{I}}: & \{\bar{E}\},\{\underline{E}\},\left\{12 \overline{C_{5}}\right\},\left\{12 \underline{C_{5}}\right\},\left\{12 \overline{C_{5}^{2}}\right\},\left\{12 \underline{C_{5}^{2}}\right\},\left\{20 \overline{C_{3}}\right\},\left\{20 \underline{C_{3}}\right\},\left\{15 \overline{C_{2}}, 15 \underline{C_{2}}\right\} . \\
\widetilde{\mathbf{T}}: & \{\bar{E}\},\{\underline{E}\},\left\{3 \overline{C_{2}}, 3 \underline{C_{2}}\right\},\left\{4 \overline{C_{3}}\right\},\left\{4 \underline{C_{3}}\right\},\left\{4 \overline{C_{3}^{2}}\right\},\left\{4 \underline{C_{3}^{2}}\right\} . \\
\widetilde{\mathbf{O}}: & \{\bar{E}\},\{\underline{E}\},\left\{6 \overline{C_{4}}\right\},\left\{6 \underline{C_{4}}\right\},\left\{3 \overline{C_{2}}, 3 \underline{C_{2}}\right\},\left\{6 \overline{C_{2}^{\prime}}, 6 \underline{C_{2}^{\prime}}\right\},\left\{8 \overline{C_{3}}\right\},\left\{8 \underline{C_{3}}\right\} . \\
\widetilde{\mathbf{O}_{\mathrm{h}}}: \quad & \{\bar{E}\},\{\underline{E}\},\left\{6 \overline{C_{4}}, 6 \underline{C_{4}}\right\},\left\{3 \overline{C_{2}}, 3 \underline{C_{2}}\right\},\left\{6 \overline{C_{2}^{\prime}}, 6 \underline{C_{2}^{\prime}}\right\},\left\{8 \overline{C_{3}}\right\},\left\{8 \underline{C_{3}}\right\}, \\
& \{\bar{I}, \underline{I}\},\left\{6 \overline{C_{4}}, 6 \underline{I I C_{4}}\right\},\left\{3 \overline{I C_{2}}, 3 \underline{I I C_{2}}\right\},\left\{6 \overline{I C_{2}^{\prime}}, 6 I \underline{C_{2}^{\prime}}\right\},\left\{8 \overline{I C_{3}}\right\},\left\{8 \underline{I C_{3}}\right\} .
\end{aligned}
$$

Supposing the $q$ th class $\mathcal{C}_{q} \quad\left(q=1, \ldots, n_{\mathcal{C}}^{\widetilde{\mathbf{P}}}\right)$ of $\widetilde{\mathbf{P}}$ consists of $h_{q}$ elements, it reads $\left\{h_{q} \bar{P}_{q}\right\}, \quad\left\{h_{q} \underline{P}_{q}\right\}$, or $\left\{\frac{h_{q}}{2} \bar{P}_{q}, \frac{h_{q}}{2} \underline{P}_{q}\right\}$.

The number of (complex) irreducible representations equals the number of classes in the group. Since all the singlevalued (complex) irreducible representations of $\mathbf{P}$, amounting to $n_{\mathcal{C}}^{\mathbf{P}}$, remain unchanged in $\widetilde{\mathbf{P}}$, we find $n_{\mathcal{C}}^{\widetilde{\mathbf{P}}}-n_{\mathcal{C}}^{\mathbf{P}}$ double-valued (complex) irreducible representations in $\widetilde{\mathbf{P}}$. When we denote the $i$ th (complex) irreducible representation of $\mathbf{P}(\widetilde{\mathbf{P}})$ by $\Xi_{i}$ $\left(\widetilde{\Xi}_{i}\right)$ and its dimensionality by $d_{\Xi_{i}}^{\mathbf{P}}\left(d_{\widetilde{\Xi}_{i}}^{\widetilde{\mathbf{P}}}\right)$, we have

$$
\begin{aligned}
& \sum_{i=1}^{n_{\mathcal{C}}^{\mathbf{I}} \equiv 5}\left(d_{\Xi_{i}}^{\mathbf{I}}\right)^{2}=g^{\mathbf{I}}=60, \\
& \sum_{i=1}^{n_{\mathcal{C}}^{\widetilde{I}} \equiv 9}\left(d_{\widetilde{\Xi}_{i}}^{\mathbf{I}}\right)^{2}=g^{\mathbf{I}}+\sum_{i=n_{\mathcal{C}}^{\mathbf{I}}+1}^{n_{\mathcal{C}}^{\widetilde{I}} \equiv 9}\left(d_{\widetilde{\Xi}_{i}}^{\mathbf{I}}\right)^{2}=g^{\widetilde{\mathbf{I}}}=120,
\end{aligned}
$$



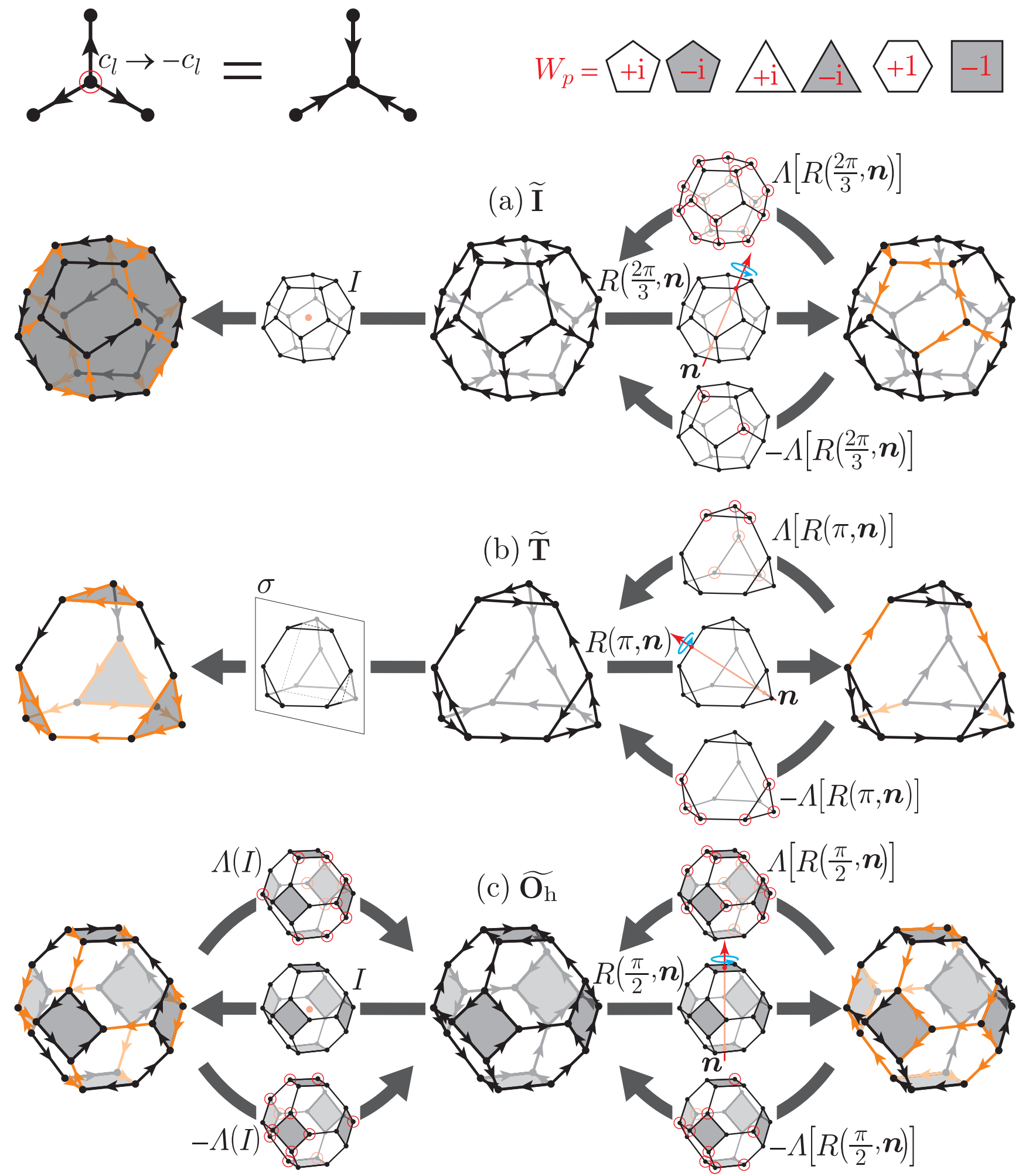

FIG. 5. Gauged rotations, (gauged) inversion, and mirror operations of gauge-ground Kitaev spin balls consisting of (a) dodecahedral, (b) truncated-tetrahedral, and (c) truncated-octahedral lattices, whose symmetry groups read $\widetilde{\mathbf{I}}, \widetilde{\mathbf{T}}$, and $\widetilde{\mathbf{O}_{\mathrm{h}}}$, respectively. Inversion $I \in \mathbf{I}_{\mathrm{h}}$ of the gauged dodecahedron and mirror operations $\sigma \in \mathbf{T}_{\mathrm{d}}$ of the gauged truncated tetrahedron can be followed by no such gauge operation as to recover the initial bond configuration. 
TABLE II. Irreducible representations of the double group $\widetilde{\mathbf{I}}$ and their characters.

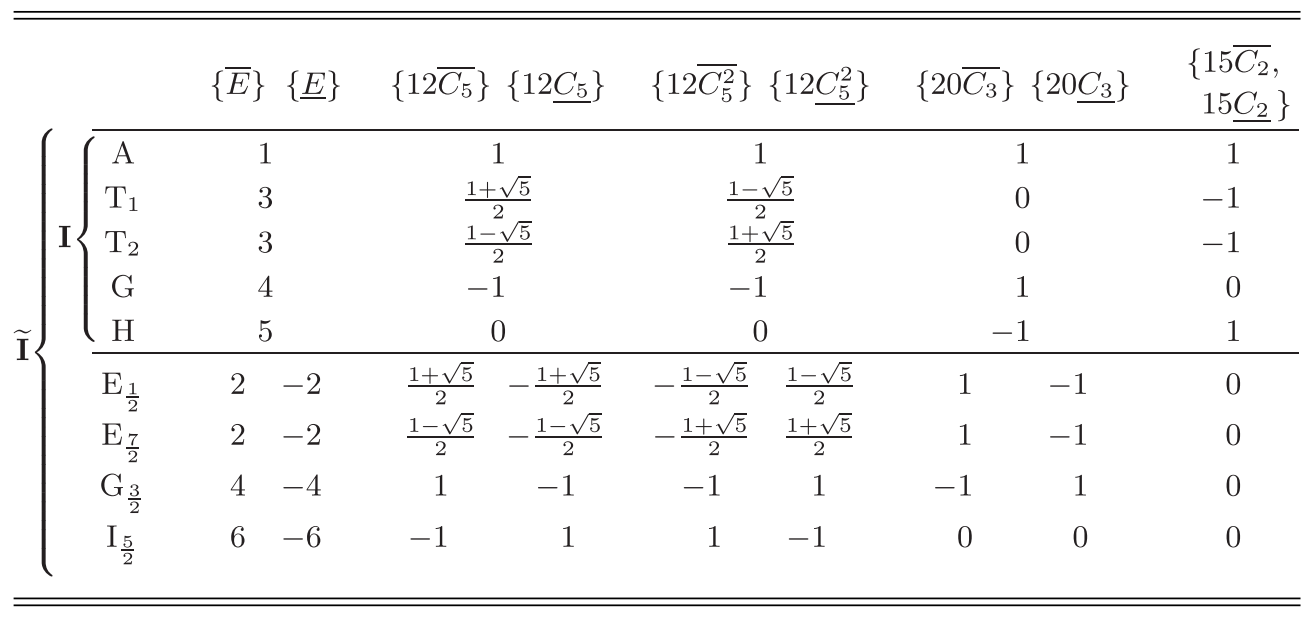

$$
\begin{aligned}
& \sum_{i=1}^{n_{\mathcal{C}}^{\mathbf{T}} \equiv 4}\left(d_{\Xi_{i}}^{\mathbf{T}}\right)^{2}=g^{\mathbf{T}}=12, \\
& \sum_{i=1}^{n_{\mathcal{C}}^{\widetilde{T}} \equiv 7}\left(d_{\widetilde{\Xi}_{i}}^{\widetilde{\mathbf{T}}}\right)^{2}=g^{\mathbf{T}}+\sum_{i=n_{\mathcal{C}}^{\mathbf{T}}+1}^{n_{\mathcal{C}}^{\widetilde{T}} \equiv 7}\left(d \widetilde{\widetilde{\Xi}}_{i}\right)^{2}=g^{\widetilde{\mathbf{T}}}=24, \\
& \sum_{i=1}^{n_{\mathcal{C}}^{\mathbf{O}} \equiv 5}\left(d_{\Xi_{i}}^{\mathbf{O}}\right)^{2}=g^{\mathbf{O}}=24 \text {, }
\end{aligned}
$$

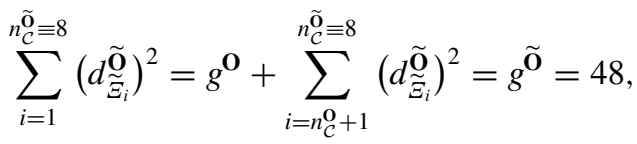

$$
\begin{aligned}
& \sum_{i=1}^{n_{\mathcal{h}}^{\mathbf{o}_{\mathrm{h}}} \equiv 10}\left(d_{\Xi_{i}}^{\mathbf{O}_{\mathrm{h}}}\right)^{2}=g^{\mathbf{O}_{\mathrm{h}}}=48
\end{aligned}
$$

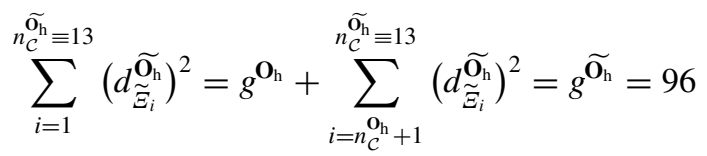

in an attempt to determine the dimensionalities of the doublevalued (complex) irreducible representations $d_{\widetilde{\Xi}_{i}}^{\widetilde{\mathbf{P}}}\left(i=n_{\mathcal{C}}^{\mathbf{P}}+\right.$ $\left.1, \ldots, n_{\mathcal{C}} \widetilde{\widetilde{\mathbf{P}}}\right)$. The characters of $\widetilde{\Xi}_{i}$ are such that

$$
\chi_{\widetilde{\Xi}_{i}}^{\widetilde{\widetilde{P}}}(\bar{P})=\chi_{\widetilde{\Xi}_{i}}^{\widetilde{\widetilde{P}}}(\underline{P}) \quad\left(i=1, \ldots, n_{\mathcal{C}}^{\mathbf{P}}\right),
$$

$$
\chi_{\widetilde{\Xi}_{i}}^{\widetilde{\widetilde{P}}}(\bar{P})=-\chi_{\widetilde{\Xi}_{i}}^{\widetilde{\widetilde{P}}}(\underline{P}) \quad\left(i=n_{\mathcal{C}}^{\mathbf{P}}+1, \ldots, n_{\mathcal{C}}^{\widetilde{\mathbf{P}}}\right)
$$

When $\bar{P}$ and $\underline{P}$ belong to the same class, i.e., $\chi_{\widetilde{\Xi}_{i}}^{\widetilde{\widetilde{P}}}(\bar{P})=\chi_{\widetilde{\Xi}_{i}}^{\widetilde{\widetilde{P}}}(\underline{P})$, we immediately find

$$
\chi_{\widetilde{\Xi}_{i}}^{\widetilde{\mathbf{P}}}(\bar{P})=\chi_{\widetilde{\Xi}_{i}}^{\widetilde{\widetilde{P}}}(\underline{P})=0 \quad\left(i=n_{\mathcal{C}}^{\mathbf{P}}+1, \ldots, n_{\mathcal{C}}^{\widetilde{\mathbf{P}}}\right) .
$$

The character orthogonality theorems of the first and second kinds read [61]

$$
\begin{gathered}
\sum_{q=1}^{h_{\mathcal{C}}^{\widetilde{\mathbf{P}}}} h_{q} \chi_{\widetilde{\Xi}_{i}}^{\widetilde{\mathbf{P}}}\left(\mathcal{C}_{q}\right)^{*} \chi_{\widetilde{\Xi}_{j}}^{\widetilde{\mathbf{P}}}\left(\mathcal{C}_{q}\right)=g^{\widetilde{\mathbf{P}}} \delta_{i j}, \\
\sum_{i=1}^{n_{\mathcal{C}}^{\widetilde{P}}} \chi_{\widetilde{\Xi}_{i}}^{\widetilde{\mathbf{P}}}\left(\mathcal{C}_{q}\right)^{*} \chi_{\widetilde{\Xi}_{i}}^{\widetilde{\mathbf{P}}}\left(\mathcal{C}_{r}\right)=\frac{g^{\widetilde{\mathbf{P}}}}{h_{q}} \delta_{q r} .
\end{gathered}
$$

When we denote the $h_{q}$ elements of $\mathcal{C}_{q}$ distinguishably as $\left\{\widetilde{P}_{q}^{(1)}, \ldots, \widetilde{P}_{q}^{\left(h_{q}\right)}\right\}$, we can define structure constants as

$$
\sum_{i=1}^{h_{q}} \widetilde{P}_{q}^{(i)} \sum_{j=1}^{h_{r}} \widetilde{P}_{r}^{(j)}=\sum_{s=1}^{n_{\mathcal{C}}^{\tilde{\mathcal{C}}}} c_{q r: s} \sum_{k=1}^{h_{s}} \widetilde{P}_{s}^{(k)}
$$

TABLE III. Irreducible representations of the double group $\widetilde{\mathbf{T}}$ and their characters.

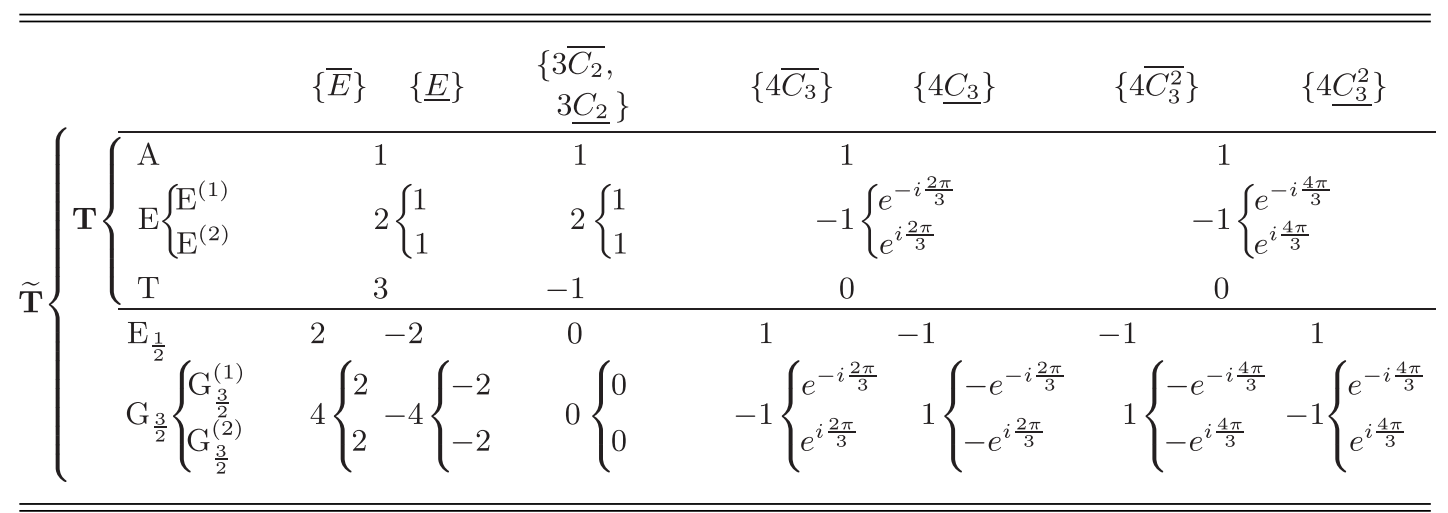


TABLE IV. Irreducible representations of the double group $\widetilde{\mathbf{O}}$ and their characters.

\begin{tabular}{|c|c|c|c|c|c|c|c|c|c|}
\hline & & $\{\bar{E}\}$ & $\{\underline{E}\}$ & $\left\{6 \overline{C_{4}}\right\}$ & $\left\{6 \underline{C_{4}}\right\}$ & $\begin{array}{l}\left\{3 \overline{C_{2}},\right. \\
\left.3 \underline{C_{2}}\right\}\end{array}$ & $\begin{array}{c}\left\{6 \overline{C_{2}^{\prime}}\right. \\
\left.\quad 6 C_{2}^{\prime}\right\}\end{array}$ & $\left\{8 \overline{C_{3}}\right\}$ & $\left\{8 \underline{C_{3}}\right\}$ \\
\hline & $\overline{A_{1}}$ & \multicolumn{2}{|c|}{1} & \multicolumn{2}{|c|}{1} & 1 & 1 & \multicolumn{2}{|c|}{1} \\
\hline & $\mathrm{A}_{2}$ & \multicolumn{2}{|c|}{1} & \multicolumn{2}{|c|}{-1} & 1 & -1 & \multicolumn{2}{|c|}{1} \\
\hline & O\{ E & \multicolumn{2}{|c|}{2} & \multicolumn{2}{|c|}{0} & 2 & 0 & \multicolumn{2}{|c|}{-1} \\
\hline & $\mathrm{T}_{1}$ & \multicolumn{2}{|c|}{3} & \multicolumn{2}{|c|}{1} & -1 & -1 & \multicolumn{2}{|c|}{0} \\
\hline $\mathrm{O}$ & $\mathrm{T}_{2}$ & \multicolumn{2}{|c|}{3} & \multicolumn{2}{|c|}{-1} & -1 & 1 & \multicolumn{2}{|c|}{0} \\
\hline & $\overline{\mathrm{E}_{\frac{1}{2}}}$ & 2 & -2 & $\sqrt{2}$ & $-\sqrt{2}$ & 0 & 0 & 1 & -1 \\
\hline & $\mathrm{E}_{\frac{5}{2}}$ & 2 & -2 & $-\sqrt{2}$ & $\sqrt{2}$ & 0 & 0 & 1 & -1 \\
\hline & $\mathrm{G}_{\frac{3}{2}}$ & 4 & -4 & 0 & 0 & 0 & 0 & -1 & 1 \\
\hline
\end{tabular}

to have another relation,

$$
h_{q} h_{r} \chi \underset{\widetilde{\Xi}_{i}}{\widetilde{\widetilde{P}}}\left(\mathcal{C}_{q}\right) \chi \underset{\widetilde{\Xi}_{i}}{\widetilde{\widetilde{P}}}\left(\mathcal{C}_{r}\right)=d \underset{\widetilde{\Xi}_{i}}{\widetilde{\mathbf{P}}} \sum_{s=1}^{n_{\mathcal{C}}^{\widetilde{\mathbf{P}}}} h_{s} c_{q r: s} \chi \underset{\widetilde{\Xi}_{i}}{\widetilde{\mathbf{P}}}\left(\mathcal{C}_{s}\right)
$$

With Eqs. (B8), (B9), (B10), and (B12) in mind, we can obtain characters of both single- and double-valued (complex) irreducible representations of any double group $\widetilde{\mathbf{P}}$, which are listed in Tables II-V, with particular emphasis on the relation between $\widetilde{\mathbf{P}}$ and $\mathbf{P}$.

\section{APPENDIX C: DIRECT-PRODUCT REPRESENTATIONS OF DOUBLE GROUPS FOR GAUGE-GROUND KITAEV POLYHEDRA}

Since Raman scattering within the LF scheme [24,53-55] is caused by spinons in pairs, we make direct-product rep- resentations out of double-valued irreducible representations of double covers $\widetilde{\mathbf{P}}$ of the corresponding point symmetry groups $\mathbf{P} \subset \mathrm{O}(3)$. Direct-product representations of a nonabelian group are not necessarily irreducible even though the constituent representations are irreducible. We take interest in spinon-geminate-excitation-relevant direct-product representations $\widetilde{\Xi}_{i} \otimes \widetilde{\Xi}_{j}\left(i, j=n_{\mathcal{C}}^{\mathbf{P}}+1, \ldots, n_{\mathcal{C}} \widetilde{\mathbf{P}}\right)$ of $\widetilde{\mathbf{P}}$, which are decomposed into single-valued irreducible representations of the corresponding point symmetry group $\mathbf{P}$,

$$
\begin{aligned}
\widetilde{\Xi}_{i} \otimes \widetilde{\Xi}_{j} & =\bigoplus_{k=1}^{n_{\mathcal{C}}^{\widetilde{\mathbf{P}}}} \widetilde{\Xi}_{k} \sum_{q=1}^{n_{\mathcal{C}}^{\widetilde{\mathbf{P}}}} \frac{h_{q}}{\underline{\underline{\mathbf{P}}}^{\widetilde{\mathbf{P}}}} \chi_{\widetilde{\Xi}_{k}}^{\widetilde{\mathbf{P}}}\left(\mathcal{C}_{q}\right)^{*} \chi \underset{\widetilde{\Xi}_{i} \otimes \widetilde{\Xi}_{j}}{\widetilde{\mathbf{P}}}\left(\mathcal{C}_{q}\right) \\
& =\bigoplus_{k=1}^{n_{\mathcal{C}}^{\mathbf{P}}} \Xi_{k} \sum_{q=1}^{n_{\mathcal{C}}^{\tilde{\mathbf{P}}}} \frac{h_{q}}{h_{\widetilde{\mathbf{P}}}} \chi_{\Xi_{k}}^{\mathbf{P}}\left(\mathcal{C}_{q}\right)^{*} \chi \underset{\widetilde{\Xi}_{i} \otimes \widetilde{\Xi}_{j}}{\widetilde{\mathbf{P}}}\left(\mathcal{C}_{q}\right),
\end{aligned}
$$

TABLE V. Irreducible representations of the double group $\widetilde{\mathbf{O}_{\mathrm{h}}}$ and their characters. Those of the direct-product group $\widetilde{\mathbf{O}} \times \mathbf{C}_{\mathrm{i}}$ are also listed.

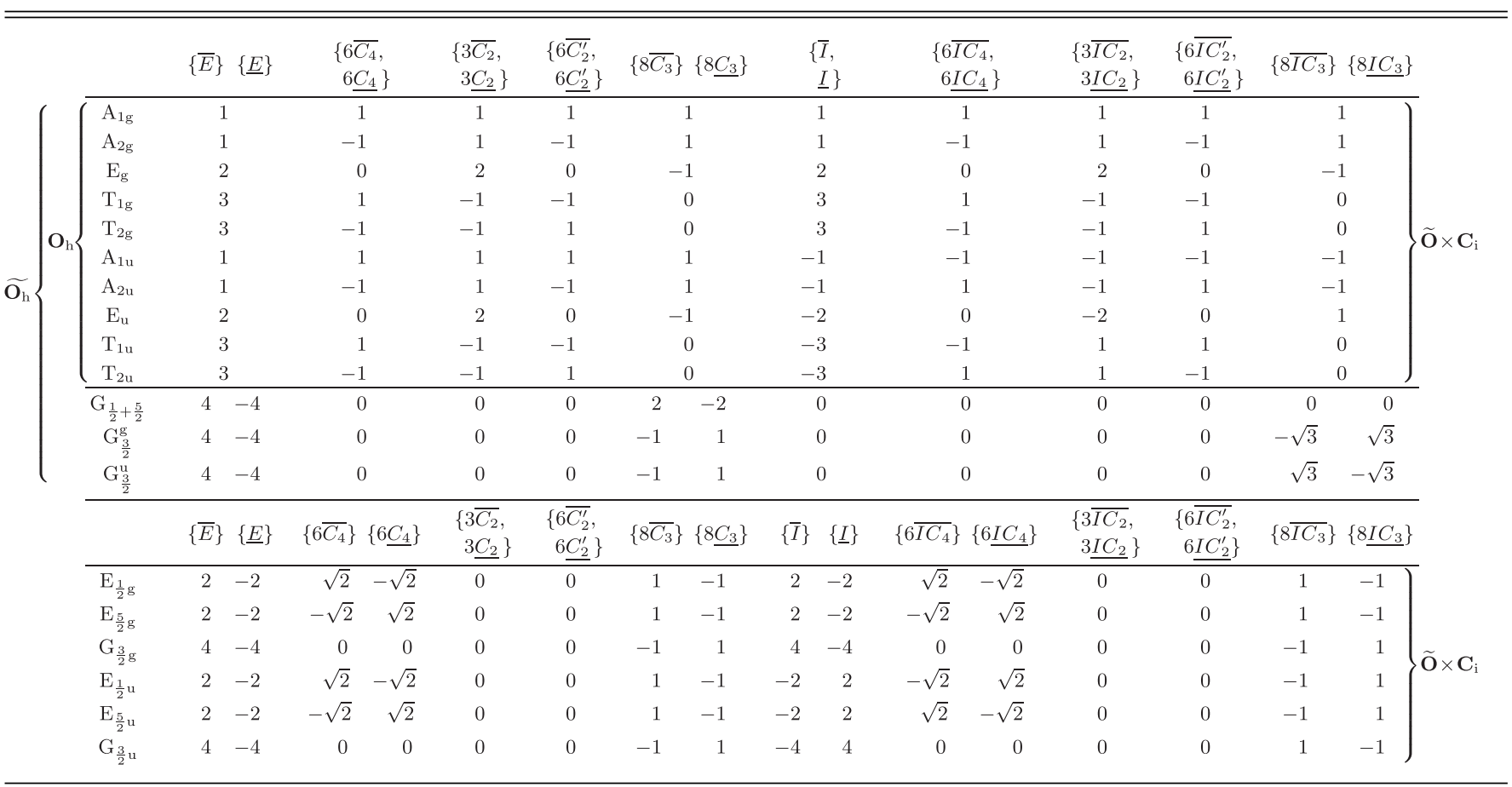


TABLE VI. Direct-product representations composed of double-valued irreducible representations of the double group $\widetilde{\mathbf{I}}$ and their characters.

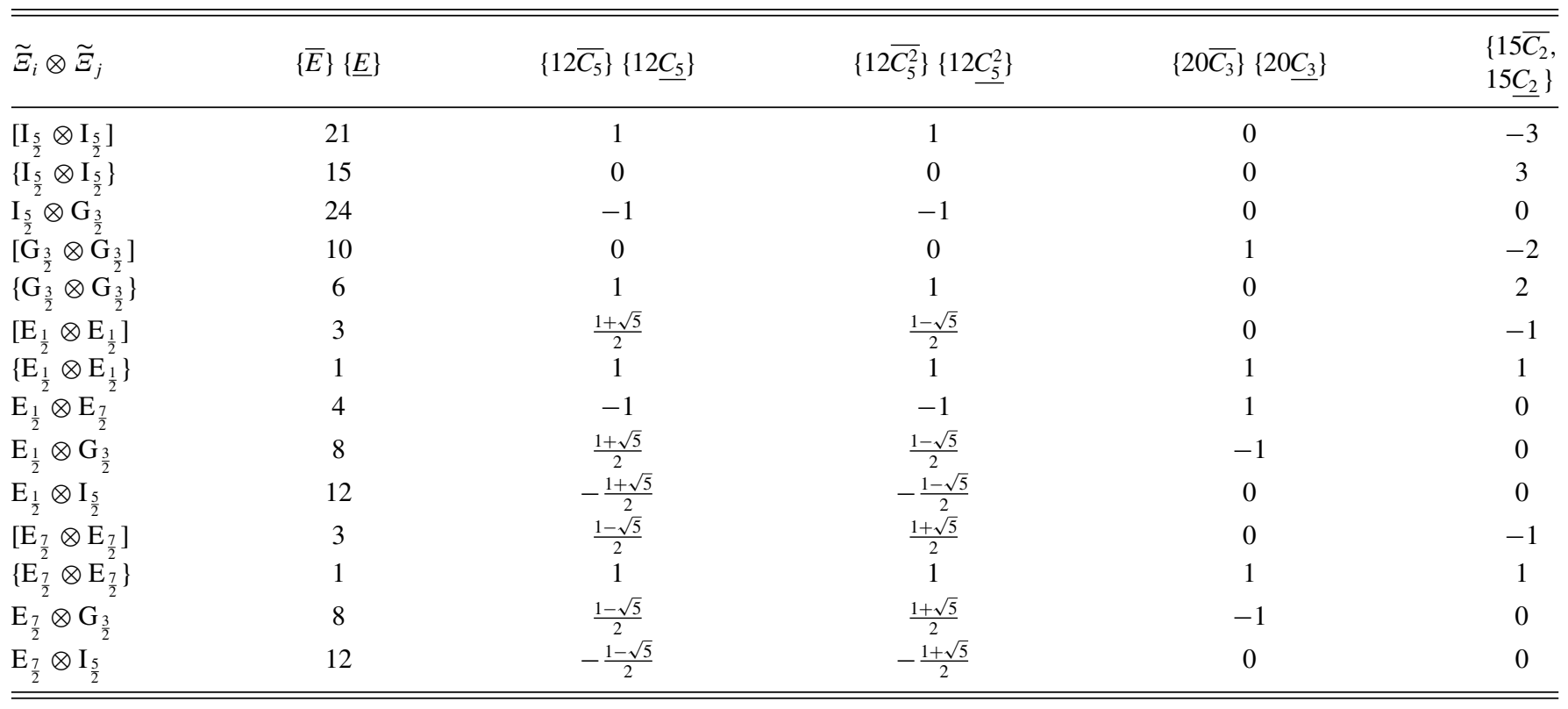

bearing in mind that

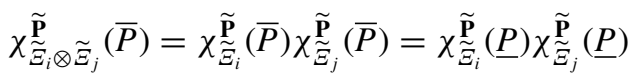

$$
\begin{aligned}
& =\chi \underset{\widetilde{\Xi}_{i} \otimes \widetilde{\Xi}_{j}}{\widetilde{\widetilde{P}}}(\underline{P}) \quad\left(i, j=n_{\mathcal{C}}^{\mathbf{P}}+1, \ldots, n_{\mathcal{C}}^{\widetilde{\mathbf{P}}}\right) .
\end{aligned}
$$

Direct-product representations made of the two same irreducible representations consist of symmetric (bosonic) and antisymmetric (fermionic) parts,

$$
\widetilde{\Xi}_{i} \otimes \widetilde{\Xi}_{i}=\left[\widetilde{\Xi}_{i} \otimes \widetilde{\Xi}_{i}\right] \oplus\left\{\widetilde{\Xi}_{i} \otimes \widetilde{\Xi}_{i}\right\}
$$

which are decomposed into symmetric and antisymmetric single-valued irreducible representations of the corresponding

TABLE VII. Direct-product representations composed of double-valued irreducible representations of the double group $\widetilde{\mathbf{T}}$ and their characters.

\begin{tabular}{lcccc}
\hline \hline$\widetilde{\Xi}_{i} \otimes \widetilde{\Xi}_{j}$ & $\{\bar{E}\}\{\underline{E}\}$ & $\begin{array}{r}\left\{3 \overline{C_{2}},\right. \\
\left.3 C_{2}\right\}\end{array}$ & $\left\{4 \overline{C_{3}}\right\}\left\{\underline{C_{3}}\right\}$ & $\left\{4 \overline{C_{3}^{2}}\right\}\left\{\underline{C_{3}^{2}}\right\}$ \\
\hline$\left[\mathrm{G}_{\frac{3}{2}}^{(2)} \otimes \mathrm{G}_{\frac{3}{2}}^{(2)}\right]$ & 3 & -1 & 0 & 0 \\
$\left\{\mathrm{G}_{\frac{3}{2}}^{(2)} \otimes \mathrm{G}_{\frac{3}{2}}^{(2)}\right\}$ & 1 & 1 & $e^{-i \frac{2}{3} \pi}$ & $e^{-i \frac{4}{3} \pi}$ \\
$\mathrm{G}_{\frac{3}{2}}^{(2)} \otimes \mathrm{E}_{\frac{1}{2}}$ & 4 & 0 & $e^{-i \frac{4}{3} \pi}$ & $e^{-i \frac{2}{3} \pi}$ \\
{$\left[\mathrm{E}_{\frac{1}{2}} \otimes \mathrm{E}_{\frac{1}{2}}\right]$} & 3 & -1 & 0 & 0 \\
$\left\{\mathrm{E}_{\frac{1}{2}} \otimes \mathrm{E}_{\frac{1}{2}}\right\}$ & 1 & 1 & 1 & 1 \\
$\mathrm{G}_{\frac{3}{2}}^{(1)} \otimes \mathrm{G}_{\frac{3}{2}}^{(2)}$ & 4 & 0 & 1 & 1 \\
$\mathrm{G}_{\frac{3}{2}}^{(1)} \otimes \mathrm{E}_{\frac{1}{2}}$ & 4 & 0 & $e^{-i \frac{2}{3} \pi}$ & $e^{-i \frac{4}{3} \pi}$ \\
{$\left[\mathrm{G}_{\frac{3}{2}}^{(1)} \otimes \mathrm{G}_{\frac{3}{2}}^{(1)}\right]$} & 3 & -1 & 0 & 0 \\
$\left\{\mathrm{G}_{\frac{3}{2}}^{(1)} \otimes \mathrm{G}_{\frac{3}{2}}^{(1)}\right\}$ & 1 & 1 & $e^{-i \frac{4}{3} \pi}$ & $e^{-i \frac{2}{3} \pi}$ \\
\hline \hline
\end{tabular}

point symmetry group $\mathbf{P}$, respectively:

$$
\begin{aligned}
& {\left[\widetilde{\Xi}_{i} \otimes \widetilde{\Xi}_{i}\right]=\bigoplus_{k=1}^{n_{\mathcal{C}}^{\mathbf{P}}}\left[\Xi_{k}\right] \sum_{q=1}^{n_{\mathcal{P}}^{\widetilde{\mathbf{P}}}} \frac{h_{q}}{g^{\widetilde{\mathbf{P}}}} \chi_{\Xi_{k}}^{\mathbf{P}}\left(\mathcal{C}_{q}\right)^{*} \chi_{\left[\widetilde{\Xi}_{i} \otimes \widetilde{\Xi}_{i}\right]}^{\widetilde{\mathbf{P}}}\left(\mathcal{C}_{q}\right),} \\
& \left\{\widetilde{\Xi}_{i} \otimes \widetilde{\Xi}_{i}\right\}=\bigoplus_{k=1}^{n_{\mathcal{C}}^{\mathbf{P}}}\left\{\Xi_{k}\right\} \sum_{q=1}^{n_{\mathcal{C}}^{\widetilde{P}}} \frac{h_{q}}{g^{\widetilde{\mathbf{P}}}} \chi_{\Xi_{k}}^{\mathbf{P}}\left(\mathcal{C}_{q}\right)^{*} \chi_{\left\{\widetilde{\Xi}_{i} \otimes \widetilde{\Xi}_{i}\right\}}^{\widetilde{\mathbf{P}}}\left(\mathcal{C}_{q}\right) .
\end{aligned}
$$

Note that characters of symmetric and antisymmetric directproduct representations are given by

$$
\begin{aligned}
& \chi_{\left[\widetilde{\Xi}_{i} \otimes \widetilde{\Xi}_{i}\right]}^{\widetilde{\mathbf{P}}}(\widetilde{P})=\frac{1}{2}\left[\chi_{\widetilde{\Xi}_{i}}^{\widetilde{\mathbf{P}}}(\widetilde{P})^{2}+\chi \underset{\widetilde{\Xi}_{i}}{\widetilde{\mathbf{P}}}\left(\widetilde{P}^{2}\right)\right], \\
& \chi_{\left\{\Xi_{i} \otimes \Xi_{i}\right\}}^{\widetilde{\mathbf{P}}}(\widetilde{P})=\frac{1}{2}\left[\chi_{\widetilde{\Xi}_{i}}^{\widetilde{\mathbf{P}}}(\widetilde{P})^{2}-\chi \underset{\widetilde{\Xi}_{i}}{\widetilde{\mathbf{P}}}\left(\widetilde{P}^{2}\right)\right] .
\end{aligned}
$$

We can obtain characters of any direct-product repre-

\begin{tabular}{|c|c|c|c|c|c|}
\hline$\widetilde{\Xi}_{i} \otimes \widetilde{\Xi}_{j}$ & $\{\bar{E}\}\{\underline{E}\}$ & $\left\{6 \overline{C_{4}}\right\}\left\{6 \underline{C_{4}}\right\}$ & $\begin{array}{l}\left\{3 \overline{C_{2}},\right. \\
\left.3 \underline{C_{2}}\right\}\end{array}$ & $\begin{array}{l}\left\{6 \overline{C_{2}^{\prime}},\right. \\
\left.6 \underline{C_{2}^{\prime}}\right\}\end{array}$ & $\left\{8 \overline{C_{3}}\right\}\left\{8 \underline{C_{3}}\right\}$ \\
\hline$\left[\mathrm{E}_{\frac{1}{2}} \otimes \mathrm{E}_{\frac{1}{2}}\right]$ & 3 & 1 & -1 & -1 & 0 \\
\hline$\left\{E_{\frac{1}{2}} \otimes E_{\frac{1}{2}}\right\}$ & 1 & 1 & 1 & 1 & 1 \\
\hline $\mathrm{E}_{\frac{1}{2}} \otimes \mathrm{E}_{\frac{5}{2}}$ & 4 & -2 & 0 & 0 & 1 \\
\hline$\left[\mathrm{E}_{\frac{5}{2}} \otimes \mathrm{E}_{\frac{5}{2}}\right]$ & 3 & 1 & -1 & -1 & 0 \\
\hline$\left\{\mathrm{E}_{\frac{5}{2}} \otimes \mathrm{E}_{\frac{5}{2}}\right\}$ & 1 & 1 & 1 & 1 & 1 \\
\hline $\mathrm{G}_{\frac{3}{2}} \otimes \mathrm{E}_{\frac{1}{2}}$ & 8 & 0 & 0 & 0 & -1 \\
\hline $\mathrm{G}_{\frac{3}{2}} \otimes \mathrm{E}_{\frac{5}{2}}$ & 8 & 0 & 0 & 0 & -1 \\
\hline$\left[\mathrm{G}_{\frac{3}{2}} \otimes \mathrm{G}_{\frac{3}{2}}\right]$ & 10 & 0 & -2 & -2 & 1 \\
\hline$\left\{\mathrm{G}_{\frac{3}{2}} \otimes \mathrm{G}_{\frac{3}{2}}\right\}$ & 6 & 0 & 2 & 2 & 0 \\
\hline
\end{tabular}
sentation using Eqs. (C6) and (C7) as well as (C2); those of our interest are listed in Tables VI-IX. Direct-product

TABLE VIII. Direct-product representations composed of double-valued irreducible representations of the double group $\widetilde{\mathbf{O}}$ and their characters. 
TABLE IX. Direct-product representations composed of double-valued irreducible representations of the double group $\widetilde{\mathbf{O}_{\mathrm{h}}}$ and their characters.

\begin{tabular}{|c|c|c|c|c|c|c|c|c|c|c|}
\hline$\widetilde{\Xi}_{i} \otimes \widetilde{\Xi}_{j}$ & $\{\bar{E}\}\{\underline{E}\}$ & $\begin{array}{l}\left\{6 \overline{C_{4}},\right. \\
\left.6 \underline{C_{4}}\right\}\end{array}$ & $\begin{array}{l}\left\{3 \overline{C_{2}},\right. \\
\left.3 \underline{C_{2}}\right\}\end{array}$ & $\begin{array}{l}\left\{6 \overline{C_{2}^{\prime}}\right. \\
\left.6 \underline{C_{2}^{\prime}}\right\}\end{array}$ & $\left\{8 \overline{C_{3}}\right\}\left\{8 \underline{C_{3}}\right\}$ & $\begin{array}{l}\{\bar{I} \\
\underline{I}\}\end{array}$ & $\begin{array}{l}\left\{6 \overline{I C_{4}},\right. \\
\left.6 I C_{4}\right\}\end{array}$ & $\begin{array}{l}\left\{3 \overline{I C_{2}},\right. \\
\left.3 I C_{2}\right\}\end{array}$ & $\begin{array}{l}\left\{6 \overline{I C_{2}^{\prime}},\right. \\
\left.6 I C_{2}^{\prime}\right\}\end{array}$ & $\left\{8 \overline{I C_{3}}\right\}\left\{8 \underline{I C_{3}}\right\}$ \\
\hline$\left[G_{\frac{1}{2}+\frac{5}{2}} \otimes G_{\frac{1}{2}+\frac{5}{2}}\right]$ & 10 & 0 & -2 & -2 & 1 & -2 & 0 & 2 & -2 & 1 \\
\hline$\left\{G_{\frac{1}{2}+\frac{5}{2}} \otimes G_{\frac{1}{2}+\frac{5}{2}}\right\}$ & 6 & 0 & 2 & 2 & 3 & 2 & 0 & -2 & 2 & -1 \\
\hline $\mathrm{G}_{\frac{3}{2}}^{\mathrm{g}} \otimes \mathrm{G}_{\frac{1}{2}+\frac{5}{2}}$ & 16 & 0 & 0 & 0 & -2 & 0 & 0 & 0 & 0 & 0 \\
\hline$\left[G_{\frac{3}{2}}^{g} \otimes G_{\frac{3}{2}}^{g}\right]$ & 10 & 0 & -2 & -2 & 1 & -2 & 0 & 2 & -2 & 1 \\
\hline$\left\{G_{\frac{3}{2}}^{\mathrm{g}} \otimes G_{\frac{3}{2}}^{\mathrm{g}}\right\}$ & 6 & 0 & 2 & 2 & 0 & 2 & 0 & -2 & 2 & 2 \\
\hline $\mathrm{G}_{\frac{3}{2}}^{\mathrm{u}} \otimes \mathrm{G}_{\frac{1}{2}+\frac{5}{2}}$ & 16 & 0 & 0 & 0 & -2 & 0 & 0 & 0 & 0 & 0 \\
\hline $\mathrm{G}_{\frac{3}{2}}^{\mathrm{g}} \otimes \mathrm{G}_{\frac{3}{2}}^{\mathrm{u}}$ & 16 & 0 & 0 & 0 & 1 & 0 & 0 & 0 & 0 & -3 \\
\hline$\left[\mathrm{G}_{\frac{3}{2}}^{\mathrm{u}} \otimes \mathrm{G}_{\frac{3}{2}}^{\mathrm{u}}\right]$ & 10 & 0 & -2 & -2 & 1 & -2 & 0 & 2 & -2 & 1 \\
\hline$\left\{G_{\frac{3}{2}}^{\mathrm{u}} \otimes \mathrm{G}_{\frac{3}{2}}^{\mathrm{u}}\right\}$ & 6 & 0 & 2 & 2 & 0 & 2 & 0 & -2 & 2 & 2 \\
\hline
\end{tabular}

representations for geminate excitations of different Majorana spinon eigenmodes are not necessarily made of different irreducible representations but may be made of the same ones. Those made of different irreducible representations can be decomposed into irreducible representations by Eq. (C1); those made of the same ones, by Eqs. (C4) and (C5). Directproduct representations for geminate excitations of degenerate Majorana spinon eigenmodes fall into the latter case. The thus-obtained decompositions into irreducible representations are all listed in Table X.

\section{APPENDIX D: POLARIZATION DEPENDENCES OF RAMAN SPECTRA}

The ground-state Raman scattering intensity of a Kitaev gauged lattice within the LF scheme [24,53-55] reads

$$
\begin{aligned}
I(\omega) & =\frac{1}{2 \pi \hbar L} \int_{-\infty}^{\infty}\left\langle 0\left|e^{\frac{i \mathscr{H} t}{\hbar}} \mathscr{R}^{\dagger} e^{-\frac{i \mathscr{H} t}{\hbar}} \mathscr{R}\right| 0\right\rangle e^{i \omega t} d t \\
& =\frac{1}{2 \pi \hbar L} \int_{-\infty}^{\infty}\left\langle 0\left|e^{\frac{i \mathscr{H} t}{\hbar}} \mathscr{R} e^{-\frac{i \mathscr{H} t}{\hbar}} \mathscr{R}\right| 0\right\rangle e^{i \omega t} d t, \\
\mathscr{R} & \equiv \sum_{\mu=x, y, z} \sum_{\nu=x, y, z} e_{\mathrm{in}}^{\mu} e_{\mathrm{sc}}^{\nu} \mathcal{R}^{\mu \nu} \equiv \boldsymbol{e}_{\mathrm{in}} \mathcal{R} \boldsymbol{e}_{\mathrm{sc}}, \\
\mathcal{R}^{\mu \nu} & \equiv-J \sum_{\lambda=x, y, z} \sum_{\langle m, n\rangle_{\lambda}} d_{m n}^{\mu} d_{m n}^{\nu} \sigma_{m}^{\lambda} \sigma_{n}^{\lambda},
\end{aligned}
$$

where $\left.\boldsymbol{e}_{\mathrm{in}} \equiv{ }^{\mathrm{t}} e_{\mathrm{in}}^{x} e_{\mathrm{in}}^{y} e_{\mathrm{in}}^{z}\right]$ and $\boldsymbol{e}_{\mathrm{sc}} \equiv\left[e_{\mathrm{sc}}^{x} e_{\mathrm{sc}}^{y} e_{\mathrm{sc}}^{z}\right]$ are the unit column vectors indicating the polarizations of incoming and outgoing photons, respectively, while $\mathcal{R} \equiv\left[\mathcal{R}^{\mu \nu}\right]$ is the matrix representation of the Raman operator in Cartesian coordinates. The matrix elements $\mathcal{R}^{\mu \nu}$ are expressed in terms of Majorana fermions and spinons:

$$
\begin{aligned}
\mathcal{R}^{\mu \nu} & =i J \sum_{\lambda=x, y, z} \sum_{\langle m, n\rangle_{\lambda}} d_{m n}^{\mu} d_{m n}^{\nu} \hat{u}_{\langle m, n\rangle_{\lambda}} c_{m} c_{n} \\
& =i J \sum_{\lambda=x, y, z} \sum_{\langle m, n\rangle_{\lambda}} \sum_{k=1}^{L / 2} \sum_{k^{\prime}=1}^{L / 2} d_{m n}^{\mu} d_{m n}^{v} \hat{u}_{\langle m, n\rangle_{\lambda}}\left[\left(\psi_{m, 2 k-1}\right.\right.
\end{aligned}
$$

$$
\begin{aligned}
& \left.\left.+i \psi_{m, 2 k}\right) \alpha_{k}^{\dagger}+\left(\psi_{m, 2 k-1}-i \psi_{m, 2 k}\right) \alpha_{k}\right]\left[\left(\psi_{n, 2 k^{\prime}-1}\right.\right. \\
& \left.\left.+i \psi_{n, 2 k^{\prime}}\right) \alpha_{k^{\prime}}^{\dagger}+\left(\psi_{n, 2 k^{\prime}-1}-i \psi_{n, 2 k^{\prime}}\right) \alpha_{k^{\prime}}\right]
\end{aligned}
$$

The LF vertex can be decomposed in terms of single-valued irreducible representations of the double group $\widetilde{\mathbf{P}}$ of the background gauged lattice, i.e., irreducible representations of the corresponding point symmetry group $\mathbf{P}$ [64-66],

$$
\mathscr{R}=\sum_{i}^{\prime} \sum_{\mu=1}^{d{ }_{\widetilde{\Xi}_{i}}^{\widetilde{\mathbf{P}}}} E_{\widetilde{\Xi}_{i}: \mu}^{\widetilde{\mathbf{P}}} \mathcal{R}_{\widetilde{\Xi}_{i}: \mu}^{\widetilde{\mathbf{P}}}=\sum_{i}^{\prime} \sum_{\mu=1}^{d_{\Xi_{i}}^{\mathbf{P}}} E_{\Xi_{i}: \mu}^{\mathbf{P}} \mathcal{R}_{\Xi_{i}: \mu}^{\mathbf{P}},
$$

where $E_{\widetilde{\Xi}_{i}: \mu}^{\widetilde{\mathbf{P}}}\left(E_{\Xi_{i}: \mu}^{\mathbf{P}}\right)$ is the $\mu$ th polarization-vector basis function for the $\widetilde{\Xi}_{i}\left(\Xi_{i}\right)$ irreducible representation of $\widetilde{\mathbf{P}}(\mathbf{P}), \mathcal{R}_{\widetilde{\Xi}_{i:}^{\widetilde{\widetilde{P}}}}^{\widetilde{\mathcal{C}}}$ $\left(\mathcal{R}_{\Xi_{i}: \mu}^{\mathbf{P}}\right)$ is the symmetry-definite LF vertex accompanying it, and $\sum_{i}^{\prime}$ runs over the $L F$-active real irreducible representations. Within the LF formulation, the nonvanishing vertices and corresponding basis functions read

$$
\begin{aligned}
E_{\mathrm{A}_{1}: 1}^{\mathbf{C}_{6 \mathrm{v}}} & =\frac{e_{\mathrm{in}}^{x} e_{\mathrm{sc}}^{x}+e_{\mathrm{in}}^{y} e_{\mathrm{sc}}^{y}}{\sqrt{2}}, \\
E_{\mathrm{E}_{2}: 1}^{\mathbf{C}_{6 \mathrm{v}}} & =\frac{e_{\mathrm{in}}^{x} e_{\mathrm{sc}}^{x}-e_{\mathrm{in}}^{y} e_{\mathrm{sc}}^{y}}{\sqrt{2}}, \\
E_{\mathrm{E}_{2}: 2}^{\mathbf{C}_{6 \mathrm{v}}} & =\frac{e_{\mathrm{in}}^{x} e_{\mathrm{sc}}^{y}+e_{\mathrm{in}}^{y} e_{\mathrm{sc}}^{x}}{\sqrt{2}}, \\
\mathcal{R}_{\mathrm{A}_{1}: 1}^{\mathbf{C}_{\mathrm{v}}} & =\frac{\mathcal{R}^{x x}+\mathcal{R}^{y y}}{\sqrt{2}}, \\
\mathcal{R}_{\mathrm{E}_{2}: 1}^{\mathbf{C}_{6 \mathrm{v}}} & =\frac{\mathcal{R}^{x x}-\mathcal{R}^{y y}}{\sqrt{2}}, \\
\mathcal{R}_{\mathrm{E}_{2}: 2}^{\mathbf{C}_{6 \mathrm{v}}} & =\frac{\mathcal{R}^{x y}+\mathcal{R}^{y x}}{\sqrt{2}}
\end{aligned}
$$

for the two-dimensional $\widetilde{\mathbf{C}_{6 \mathrm{v}}}$ gauged honeycomb and

$$
\begin{aligned}
& E_{\mathrm{A}: 1}^{\mathbf{I}}=E_{\mathrm{A}: 1}^{\mathbf{T}}=E_{\mathrm{A}_{\mathrm{lg}}: 1}^{\mathbf{O}_{\mathrm{h}}}=\frac{e_{\mathrm{in}}^{x} e_{\mathrm{sc}}^{x}+e_{\mathrm{in}}^{y} e_{\mathrm{sc}}^{y}+e_{\mathrm{in}}^{z} e_{\mathrm{sc}}^{z}}{\sqrt{3}}, \\
& E_{\mathrm{H}: 1}^{\mathbf{I}}=E_{\mathrm{E}: 1}^{\mathbf{T}}=E_{\mathrm{E}_{\mathrm{g}}: 1}^{\mathbf{O}_{\mathrm{h}}}=\frac{2 e_{\mathrm{in}}^{z} e_{\mathrm{sc}}^{z}-e_{\mathrm{in}}^{x} e_{\mathrm{sc}}^{x}-e_{\mathrm{in}}^{y} e_{\mathrm{sc}}^{y}}{\sqrt{6}},
\end{aligned}
$$


TABLE X. Direct-product representations composed of double-valued irreducible representations $\widetilde{\Xi}_{i} \otimes \widetilde{\Xi}_{j}\left(i, j=n_{\mathcal{C}}^{\mathbf{P}}+1, \ldots, n_{\mathcal{C}}^{\widetilde{\mathbf{P}}}\right)$ and their decompositions into single-valued irreducible representations $\widetilde{\Xi}_{k}\left(k=1, \ldots, n_{\mathcal{C}}^{\mathbf{P}}\right)$, which are doubly or singly underlined when they are relevant to inelastic (Raman) or elastic (Rayleigh) light scatterings, respectively, for various double groups $\widetilde{\mathbf{P}}$. Note that $\widetilde{\Xi_{k}}$ of $\widetilde{\mathbf{P}}$ is nothing but $\Xi_{k}$ of $\mathbf{P}$.

\begin{tabular}{|c|c|c|}
\hline$\widetilde{\mathbf{P}}$ & $\widetilde{\Xi}_{i} \otimes \widetilde{\Xi}_{j}$ & $\bigoplus_{k} \widetilde{\Xi}_{k}=\bigoplus_{k} \Xi_{k}$ \\
\hline \multirow[t]{10}{*}{$\widetilde{\mathbf{I}}$} & $\mathrm{I}_{\frac{5}{2}} \otimes \mathrm{I}_{\frac{5}{2}}$ & $\{\underline{\mathrm{A}}\} \oplus 2\left[\mathrm{~T}_{1}\right] \oplus 2\left[\mathrm{~T}_{2}\right] \oplus[\mathrm{G}] \oplus\{\mathrm{G}\} \oplus[\underline{\underline{\mathrm{H}}}] \oplus 2\{\underline{\underline{\mathrm{H}}}\}$ \\
\hline & $\mathrm{I}_{\frac{5}{2}} \otimes \mathrm{G}_{\frac{3}{2}}$ & $\mathrm{~T}_{1} \oplus \mathrm{T}_{2} \oplus 2 \mathrm{G} \oplus 2 \underline{\underline{H}}$ \\
\hline & $\mathrm{G}_{\frac{3}{2}} \otimes \mathrm{G}_{\frac{3}{2}}$ & $\{\underline{\mathrm{A}}\} \oplus\left[\mathrm{T}_{1}\right] \oplus\left[\mathrm{T}_{2}\right] \oplus[\mathrm{G}] \oplus\{\underline{\underline{\mathrm{H}}}\}$ \\
\hline & $\mathrm{E}_{\frac{1}{2}} \otimes \mathrm{E}_{\frac{1}{2}}$ & $\{\underline{\mathrm{A}}\} \oplus\left[\mathrm{T}_{1}\right]$ \\
\hline & $\mathrm{E}_{\frac{1}{2}} \otimes \mathrm{E}_{\frac{7}{2}}$ & $\mathrm{G}$ \\
\hline & $\mathrm{E}_{\frac{1}{2}} \otimes \mathrm{G}_{\frac{3}{2}}$ & $\mathrm{~T}_{1} \oplus \underline{\underline{\mathrm{H}}}$ \\
\hline & $\mathrm{E}_{\frac{1}{2}} \otimes \mathrm{I}_{\frac{5}{2}}$ & $\mathrm{~T}_{2} \oplus \mathrm{G} \oplus \underline{\underline{H}}$ \\
\hline & $\mathrm{E}_{\frac{7}{2}} \otimes \mathrm{E}_{\frac{7}{2}}$ & $\{\underline{\mathrm{A}}\} \oplus\left[\mathrm{T}_{2}\right]$ \\
\hline & $\mathrm{E}_{\frac{7}{2}} \otimes \mathrm{G}_{\frac{3}{2}}$ & $\mathrm{~T}_{2} \oplus \underline{\underline{\mathrm{H}}}$ \\
\hline & $\mathrm{E}_{\frac{7}{2}} \otimes \mathrm{I}_{\frac{5}{2}}$ & $\mathrm{~T}_{1} \oplus \mathrm{G} \oplus \underline{\underline{\underline{H}}}$ \\
\hline \multirow[t]{6}{*}{$\widetilde{\mathbf{T}}$} & $\mathrm{G}_{\frac{3}{2}}^{(2)} \otimes \mathrm{G}_{\frac{3}{2}}^{(2)}$ & $\left\{\underline{\underline{\mathrm{E}^{(1)}}}\right\} \oplus[\underline{\underline{T}}]$ \\
\hline & $\mathrm{G}_{\frac{3}{2}}^{(2)} \otimes \mathrm{E}_{\frac{1}{2}}$ & $\underline{\underline{\mathrm{E}^{(2)}}} \oplus \underline{\underline{\mathrm{T}}}$ \\
\hline & $\mathrm{E}_{\frac{1}{2}} \otimes \mathrm{E}_{\frac{1}{2}}$ & $\{\underline{\mathrm{A}}\} \oplus[\underline{\mathrm{T}}]$ \\
\hline & $\mathrm{G}_{\frac{3}{2}}^{(1)} \otimes \mathrm{G}_{\frac{3}{2}}^{(2)}$ & $\underline{\mathrm{A}} \oplus \underline{\underline{T}}$ \\
\hline & $\mathrm{G}_{\frac{3}{2}}^{(1)} \otimes \mathrm{E}_{\frac{1}{2}}$ & $\underline{\underline{\mathrm{E}^{(1)}}} \oplus \underline{\underline{T}}$ \\
\hline & $\mathrm{G}_{\frac{3}{2}}^{(1)} \otimes \mathrm{G}_{\frac{3}{2}}^{(1)}$ & $\left\{\underline{\underline{\mathrm{E}^{(2)}}}\right\} \oplus[\underline{\underline{T}}]$ \\
\hline \multirow[t]{6}{*}{$\widetilde{\mathbf{O}}$} & $\mathrm{E}_{\frac{1}{2}} \otimes \mathrm{E}_{\frac{1}{2}}$ & $\left\{\underline{\mathrm{A}_{1}}\right\} \oplus\left[\mathrm{T}_{1}\right]$ \\
\hline & $\mathrm{E}_{\frac{1}{2}} \otimes \mathrm{E}_{\frac{5}{2}}$ & $\mathrm{~A}_{2} \oplus \underline{\underline{\underline{\mathrm{T}_{2}}}}$ \\
\hline & $\mathrm{E}_{\frac{5}{2}} \otimes \mathrm{E}_{\frac{5}{2}}$ & $\left\{\underline{\mathrm{A}_{1}}\right\} \oplus\left[\mathrm{T}_{1}\right]$ \\
\hline & $\mathrm{G}_{\frac{3}{2}} \otimes \mathrm{E}_{\frac{1}{2}}$ & $\underline{\underline{E}} \oplus \mathrm{T}_{1} \oplus \underline{\underline{\underline{\mathrm{T}_{2}}}}$ \\
\hline & $\mathrm{G}_{\frac{3}{2}} \otimes \mathrm{E}_{\frac{5}{2}}$ & $\underline{\underline{E}} \oplus \mathrm{T}_{1} \oplus \underline{\underline{\underline{\mathrm{T}_{2}}}}$ \\
\hline & $\mathrm{G}_{\frac{3}{2}} \otimes \mathrm{G}_{\frac{3}{2}}$ & $\left\{\underline{\mathrm{A}_{1}}\right\} \oplus\left[\mathrm{A}_{2}\right] \oplus\{\underline{\underline{\mathrm{E}}}\} \oplus 2\left[\mathrm{~T}_{1}\right] \oplus\left[\underline{\underline{\mathrm{T}_{2}}}\right] \oplus\left\{\underline{\underline{\mathrm{T}_{2}}}\right\}$ \\
\hline \multirow[t]{6}{*}{$\widetilde{\mathbf{O}_{\mathrm{h}}}$} & $\mathrm{G}_{\frac{1}{2}+\frac{5}{2}} \otimes \mathrm{G}_{\frac{1}{2}+\frac{5}{2}}$ & $\left\{\underline{\mathrm{A}_{1 \mathrm{~g}}}\right\} \oplus\left\{\mathrm{A}_{1 \mathrm{u}}\right\} \oplus\left[\mathrm{A}_{2 \mathrm{~g}}\right] \oplus\left\{\mathrm{A}_{2 \mathrm{u}}\right\} \oplus\left[\mathrm{T}_{1 \mathrm{~g}}\right] \oplus\left[\mathrm{T}_{1 \mathrm{u}}\right] \oplus\left\{\underline{\underline{\mathrm{T}_{2 \mathrm{~g}}}}\right\} \oplus\left[\mathrm{T}_{2 \mathrm{u}}\right]$ \\
\hline & $\mathrm{G}_{\frac{3}{2}}^{\mathrm{g}} \otimes \mathrm{G}_{\frac{1}{2}+\frac{5}{2}}$ & $\underline{\underline{\mathrm{E}_{\mathrm{g}}}} \oplus \mathrm{E}_{\mathrm{u}} \oplus \mathrm{T}_{1 \mathrm{~g}} \oplus \mathrm{T}_{1 \mathrm{u}} \oplus \underline{\underline{\mathrm{T}_{2 \mathrm{~g}}}} \oplus \mathrm{T}_{2 \mathrm{u}}$ \\
\hline & $\mathrm{G}_{\frac{3}{2}}^{\mathrm{g}} \otimes \mathrm{G}_{\frac{3}{2}}^{\mathrm{g}}$ & $\left\{\underline{\mathrm{A}_{1 \mathrm{~g}}}\right\} \oplus\left[\mathrm{A}_{2 \mathrm{~g}}\right] \oplus\left\{\mathrm{E}_{\mathrm{u}}\right\} \oplus\left[\mathrm{T}_{1 \mathrm{~g}}\right] \oplus\left[\mathrm{T}_{1 \mathrm{u}}\right] \oplus\left\{\underline{\underline{\mathrm{T}_{2 \mathrm{~g}}}}\right\} \oplus\left[\mathrm{T}_{2 \mathrm{u}}\right]$ \\
\hline & $\mathrm{G}_{\frac{3}{2}}^{\mathrm{u}} \otimes \mathrm{G}_{\frac{1}{2}+\frac{5}{2}}$ & $\underline{\underline{\mathrm{E}_{\mathrm{g}}}} \oplus \mathrm{E}_{\mathrm{u}} \oplus \mathrm{T}_{1 \mathrm{~g}} \oplus \mathrm{T}_{1 \mathrm{u}} \oplus \underline{\underline{\underline{\mathrm{T}_{2 \mathrm{~g}}}}} \oplus \mathrm{T}_{2 \mathrm{u}}$ \\
\hline & $\mathrm{G}_{\frac{3}{2}}^{\mathrm{g}} \otimes \mathrm{G}_{\frac{3}{2}}^{\mathrm{u}}$ & $\overline{\mathrm{A}}_{1 \mathrm{u}} \oplus \mathrm{A}_{2 \mathrm{u}} \oplus \underline{\underline{\underline{\mathrm{E}_{\mathrm{g}}}}} \oplus \mathrm{T}_{1 \mathrm{~g}} \overline{\overline{ }}_{1 \mathrm{u}} \oplus \underline{\underline{\mathrm{T}_{2 \mathrm{~g}}}} \oplus \mathrm{T}_{2 \mathrm{u}}$ \\
\hline & $\mathrm{G}_{\frac{3}{2}}^{\mathrm{u}} \otimes \mathrm{G}_{\frac{3}{2}}^{\mathrm{u}}$ & $\left\{\underline{\mathrm{A}_{1 \mathrm{~g}}}\right\} \oplus\left[\mathrm{A}_{2 \mathrm{~g}}\right] \oplus\left\{\mathrm{E}_{\mathrm{u}}\right\} \oplus\left[\mathrm{T}_{1 \mathrm{~g}}\right] \oplus\left[\mathrm{T}_{1 \mathrm{u}}\right] \oplus\left\{\underline{\underline{\mathrm{T}_{2 \mathrm{~g}}}}\right\} \oplus\left[\mathrm{T}_{2 \mathrm{u}}\right]$ \\
\hline
\end{tabular}

$$
\begin{aligned}
& E_{\mathrm{H}: 2}^{\mathbf{I}}=E_{\mathrm{E}: 2}^{\mathbf{T}}=E_{\mathrm{E}_{\mathrm{g}}: 2}^{\mathbf{O}_{\mathrm{h}}}=\frac{e_{\mathrm{in}}^{x} e_{\mathrm{sc}}^{x}-e_{\mathrm{in}}^{y} e_{\mathrm{sc}}^{y}}{\sqrt{2}}, \\
& E_{\mathrm{H}: 3}^{\mathbf{I}}=E_{\mathrm{T}: 1}^{\mathbf{T}}=E_{\mathrm{T}_{\mathrm{g} g}: 1}^{\mathbf{O}_{\mathrm{h}}}=\frac{e_{\mathrm{in}}^{x} e_{\mathrm{sc}}^{y}+e_{\mathrm{in}}^{y} e_{\mathrm{sc}}^{x}}{\sqrt{2}} \\
& E_{\mathrm{H}: 4}^{\mathbf{I}}=E_{\mathrm{T}: 2}^{\mathbf{T}}=E_{\mathrm{T}_{2 \mathrm{~g}}: 2}^{\mathbf{O}_{\mathrm{h}}}=\frac{e_{\mathrm{in}}^{y} e_{\mathrm{sc}}^{z}+e_{\mathrm{in}}^{z} e_{\mathrm{sc}}^{y}}{\sqrt{2}}, \\
& E_{\mathrm{H}: 5}^{\mathbf{I}}=E_{\mathrm{T}: 3}^{\mathbf{T}}=E_{\mathrm{T}_{2 \mathrm{~g}}: 3}^{\mathbf{O}_{\mathrm{h}}}=\frac{e_{\mathrm{in}}^{z} e_{\mathrm{sc}}^{x}+e_{\mathrm{in}}^{x} e_{\mathrm{sc}}^{z}}{\sqrt{2}}, \\
& \mathcal{R}_{\mathrm{A}: 1}^{\mathbf{I}}=\mathcal{R}_{\mathrm{A}: 1}^{\mathbf{T}}=\mathcal{R}_{\mathrm{A}_{\mathrm{h} g}: 1}^{\mathbf{O}_{\mathrm{h}}}=\frac{\mathcal{R}^{x x}+\mathcal{R}^{y y}+\mathcal{R}^{z z}}{\sqrt{3}},
\end{aligned}
$$

$$
\begin{aligned}
& \mathcal{R}_{\mathrm{H}: 1}^{\mathbf{I}}=\mathcal{R}_{\mathrm{E}: 1}^{\mathbf{T}}=\mathcal{R}_{\mathrm{E}_{\mathrm{g}}: 1}^{\mathbf{O}_{\mathrm{g}}}=\frac{2 \mathcal{R}^{z z}-\mathcal{R}^{x x}-\mathcal{R}^{y y}}{\sqrt{6}}, \\
& \mathcal{R}_{\mathrm{H}: 2}^{\mathbf{I}}=\mathcal{R}_{\mathrm{E}: 2}^{\mathbf{T}}=\mathcal{R}_{\mathrm{E}_{\mathrm{g}}: 2}^{\mathbf{O}_{\mathrm{h}}}=\frac{\mathcal{R}^{x x}-\mathcal{R}^{y y}}{\sqrt{2}}, \\
& \mathcal{R}_{\mathrm{H}: 3}^{\mathbf{I}}=\mathcal{R}_{\mathrm{T}: 1}^{\mathbf{T}}=\mathcal{R}_{\mathrm{T}_{2 \mathrm{~g}}: 1}^{\mathbf{O}_{\mathrm{h}}}=\frac{\mathcal{R}^{x y}+\mathcal{R}^{y x}}{\sqrt{2}}, \\
& \mathcal{R}_{\mathrm{H}: 4}^{\mathbf{I}}=\mathcal{R}_{\mathrm{T}: 2}^{\mathbf{T}}=\mathcal{R}_{\mathrm{T}_{2 \mathrm{~g}}: 2}^{\mathbf{O}_{\mathrm{h}}}=\frac{\mathcal{R}^{y z}+\mathcal{R}^{z y}}{\sqrt{2}}, \\
& \mathcal{R}_{\mathrm{H}: 5}^{\mathbf{I}}=\mathcal{R}_{\mathrm{T}: 3}^{\mathbf{T}}=\mathcal{R}_{\mathrm{T}_{2 \mathrm{~g}}: 3}^{\mathbf{O}_{\mathrm{h}}}=\frac{\mathcal{R}^{z x}+\mathcal{R}^{x z}}{\sqrt{2}}
\end{aligned}
$$


for the $\widetilde{\mathbf{I}}, \widetilde{\mathbf{T}}$, and $\widetilde{\mathbf{O}_{\mathrm{h}}}$ gauged polyhedra, where $\mathcal{R}_{\mathrm{A}_{1}: 1}^{\mathbf{C}_{6 \mathrm{v}}}$ and $\mathcal{R}_{\mathrm{A}: 1}^{\mathbf{I}}=\mathcal{R}_{\mathrm{A}: 1}^{\mathbf{T}}=\mathcal{R}_{\mathrm{A}_{\mathrm{h} g}: 1}^{\mathbf{O}_{\mathrm{h}}}$, belonging to the identity representations in two and three dimensions, respectively, all commute with the corresponding Hamiltonians to contribute merely to elastic (Rayleigh) scattering.
Decomposing the Raman operator into irreducible representations (D3) and taking account of their orthogonality (21), we write the Raman scattering intensity as

$$
\begin{aligned}
I(\omega) & =\sum_{i}^{\prime} \sum_{j}^{\prime} \sum_{\mu=1}^{d_{\Xi_{i}}^{\mathbf{P}}} \sum_{\nu=1}^{d_{\Xi_{j}}^{\mathbf{P}}} E_{\Xi_{i}: \mu}^{\mathbf{P}} E_{\Xi_{j}: \nu}^{\mathbf{P}} \int_{-\infty}^{\infty} \frac{d t e^{i \omega t}}{2 \pi \hbar L}\left\langle 0\left|e^{\frac{i \mathscr{H} t_{t}}{\hbar}} \mathcal{R}_{\Xi_{i}: \mu}^{\mathbf{P}} e^{-\frac{i \mathscr{H} e_{t}}{\hbar}} \mathcal{R}_{\Xi_{j}: \nu}^{\mathbf{P}}\right| 0\right\rangle \\
& =\sum_{i}^{\prime} \sum_{\mu=1}^{d_{\Xi_{i}}^{\mathbf{P}}}\left(E_{\Xi_{i}: \mu}^{\mathbf{P}}\right)^{2} \int_{-\infty}^{\infty} \frac{d t e^{i \omega t}}{2 \pi \hbar L}\left\langle 0\left|e^{\frac{i \mathscr{\mathcal { E }} t}{\hbar}} \mathcal{R}_{\Xi_{i}: \mu}^{\mathbf{P}} e^{-\frac{i \mathscr{H} t}{\hbar}} \mathcal{R}_{\Xi_{i}: \mu}^{\mathbf{P}}\right| 0\right\rangle \equiv \sum_{i}^{\prime} \sum_{\mu=1}^{d_{\Xi_{i}}^{\mathbf{P}}}\left(E_{\Xi_{i}: \mu}^{\mathbf{P}}\right)^{2} I_{\Xi_{i}: \mu}^{\mathbf{P}}(\omega) .
\end{aligned}
$$

We write the Raman vertices in Cartesian coordinates (D5) and then in terms of spinon operators (D2). Having in mind that $\alpha_{k}|0\rangle=0$ and discarding Rayleigh terms, we can express $I(\omega)$ by Fermi's golden rule,

$$
\begin{aligned}
& I(\omega)=\sum_{i}^{\prime} \sum_{\mu=1}^{d_{\Xi_{i}}^{\mathbf{P}}}\left(E_{\Xi_{i}: \mu}^{\mathbf{P}}\right)^{2} \int_{-\infty}^{\infty} \frac{d t e^{i \omega t}}{2 \pi \hbar L} \sum_{q=0}^{2^{\frac{L}{2}+1}-1} \sum_{\kappa=0}^{2^{\frac{L}{2}-1}-1}\left\langle 0\left|e^{\frac{i \mathscr{F} \mathcal{F}_{t}}{\hbar}} \mathcal{R}_{\Xi_{i}: \mu}^{\mathbf{P}} e^{-\frac{i \mathscr{H} \ell_{t}}{\hbar}}\right|\left\{n_{k}\right\}\right\rangle_{\kappa} \otimes\left|\left\{W_{p}\right\}\right\rangle_{q q}\left\langle\left\{W_{p}\right\}\right| \otimes_{\kappa}\left\langle\left\{n_{k}\right\}\left|\mathcal{R}_{\Xi_{i}: \mu}^{\mathbf{P}}\right| 0\right\rangle \\
& =\frac{1}{L} \sum_{i}^{\prime} \sum_{\mu=1}^{d_{\Xi_{i}}^{\mathbf{P}}}\left(E_{\Xi_{i}: \mu}^{\mathbf{P}}\right)^{2} \frac{1}{2 \pi \hbar} \sum_{1=k^{\prime}<k^{\prime \prime}=\frac{L}{2}} \int_{-\infty}^{\infty} e^{i\left(\omega-\frac{\varepsilon_{k^{\prime}}}{\hbar}-\frac{\varepsilon_{k^{\prime \prime}}}{\hbar}\right) t} d t \sum_{q=0}^{2^{\frac{L}{2}+1}-1}{ }_{0}\left\langle\left\{W_{p}\right\} \mid\left\{W_{p}\right\}\right\rangle_{q q}\left\langle\left\{W_{p}\right\} \mid\left\{W_{p}\right\}\right\rangle_{0} \\
& \times{ }_{0}\left\langle\left\{n_{k}\right\}\left|\mathcal{R}_{\Xi_{i}: \mu}^{\mathbf{P}}\right|_{\left\{u_{(m, n)_{\lambda}}\right\}_{0(r)}} \alpha_{k^{\prime \prime}}^{\dagger} q_{k^{\prime}}^{\dagger} \mid\left\{n_{k}\right\}\right\rangle_{00}\left\langle\left\{n_{k}\right\}\left|\alpha_{k^{\prime}} \alpha_{k^{\prime \prime}} \mathcal{R}_{\Xi_{i:}: \mu}^{\mathbf{P}}\right|_{\left\{u_{(m, n)_{\lambda}}\right\}_{0(r)}} \mid\left\{n_{k}\right\}\right\rangle_{0} \\
& =\frac{1}{L} \sum_{i}^{\prime} \sum_{\mu=1}^{d_{\Xi_{i}}^{\mathbf{P}}}\left(E_{\Xi_{i}: \mu}^{\mathbf{P}}\right)^{2} \sum_{1=k<k^{\prime}=\frac{L}{2}}\left|\left\langle 0\left|\alpha_{k} \alpha_{k^{\prime}} \mathcal{R}_{\Xi_{i}: \mu}^{\mathbf{P}}\right| 0\right\rangle\right|^{2} \delta\left(\hbar \omega-\varepsilon_{k}-\varepsilon_{k^{\prime}}\right)
\end{aligned}
$$

where $\left.\mathcal{R}_{\Xi_{i}: \mu}^{\mathbf{P}}\right|_{\left\{u_{(m, n)}\right\}_{0}(r)}$ are the gauge-ground LF vertices.

The spectral degeneracy within each multidimensional irreducible representation [62] is the case with Kitaev spin balls as well. Considering the QSL ground state (9) is invariant under every symmetry operation $P \in \mathbf{P}$, the Raman response with $\mathcal{P} \boldsymbol{e}_{\text {in }} \equiv \tilde{\boldsymbol{e}}_{\text {in }}$ and $\mathcal{P} \boldsymbol{e}_{\mathrm{sc}} \equiv \tilde{\boldsymbol{e}}_{\mathrm{sc}}$, which we denote by $\tilde{I}(\omega)$, should remain the same as $I(\omega)$ with $\boldsymbol{e}_{\text {in }}$ and $\boldsymbol{e}_{\mathrm{sc}}$, where we denote the matrix representation in Cartesian coordinates for the point symmetry operation $P$ by $\mathcal{P}$. With Eq. (D1) in mind, a point symmetry operation of the Raman operator reads

$$
\tilde{\boldsymbol{e}}_{\mathrm{in}} \mathcal{R} \tilde{\boldsymbol{e}}_{\mathrm{sc}} \equiv \widetilde{\mathscr{R}}=\sum_{\mu, v=x, y, z} \sum_{\mu^{\prime}, \nu^{\prime}=x, y, z} e_{\mathrm{in}}^{\mu \mathrm{t}} \mathcal{P}^{\mu \mu^{\prime}} \mathcal{R}^{\mu^{\prime} v^{\prime}} \mathcal{P}^{\nu^{\prime} v} e_{\mathrm{sc}}^{\nu} \equiv \sum_{\mu, \nu=x, y, z} e_{\mathrm{in}}^{\mu} \widetilde{\mathcal{R}}^{\mu \nu}(P) e_{\mathrm{sc}}^{v} \equiv{ }^{\mathrm{t}} \boldsymbol{e}_{\mathrm{in}} \widetilde{\mathcal{R}}(P) \boldsymbol{e}_{\mathrm{sc}}
$$

and therefore, we have the intensity

$$
\begin{aligned}
\tilde{I}(\omega) & =\int_{-\infty}^{\infty} \frac{d t e^{i \omega t}}{2 \pi \hbar L}\left\langle 0\left|e^{\frac{i \mathscr{H} t}{\hbar}} \widetilde{\mathscr{R}} e^{-\frac{i \mathscr{H} t}{\hbar}} \widetilde{\mathscr{R}}\right| 0\right\rangle=\sum_{i}^{\prime} \sum_{\mu=1}^{d_{\Xi_{i}}^{\mathbf{P}}}\left(E_{\Xi_{i}: \mu}^{\mathbf{P}}\right)^{2} \int_{-\infty}^{\infty} \frac{d t e^{i \omega t}}{2 \pi \hbar L}\left\langle 0\left|e^{\frac{i \mathscr{H} t}{\hbar}} \widetilde{\mathcal{R}}_{\Xi_{i}: \mu}^{\mathbf{P}}(P) e^{-\frac{i \mathscr{H} t}{\hbar}} \widetilde{\mathcal{R}}_{\Xi_{i}: \mu}^{\mathbf{P}}(P)\right| 0\right\rangle \\
& \equiv \sum_{i}^{\prime} \sum_{\mu=1}^{d_{\Xi_{i}}^{\mathbf{P}}}\left(E_{\Xi_{i}: \mu}^{\mathbf{P}}\right)^{2} \tilde{I}_{\Xi_{i}: \mu}^{\mathbf{P}}(\omega)=\sum_{i}^{\prime} \sum_{\mu=1}^{d_{\Xi_{i}}^{\mathbf{P}}}\left(E_{\Xi_{i}: \mu}^{\mathbf{P}}\right)^{2} I_{\Xi_{i}: \mu}^{\mathbf{P}}(\omega) .
\end{aligned}
$$

Arbitrary polarization vectors $\boldsymbol{e}_{\text {in }}$ and $\boldsymbol{e}_{\text {sc }}$ yield arbitrary coefficients $\left(E_{\Xi_{i: \mu}}^{\mathbf{P}}\right)^{2}$ and therefore demand that $\tilde{I}_{\Xi_{i}: \mu}^{\mathbf{P}}(\omega)=I_{\Xi_{i}: \mu}^{\mathbf{P}}(\omega)$ for every Raman-active mode $\Xi_{i}: \mu$. It is instructive to review the Raman-active $\mathrm{E}_{2}$ symmetry species of the $\mathbf{C}_{6 \mathrm{v}}$ honeycomb lattice [62] on the $x y$ plane. The threefold rotation about the $z$ axis of the polarization vectors reads converting the Raman operator into

$$
C_{3(z)} \mathcal{R} \equiv \widetilde{\mathcal{R}}\left(C_{3(z)}\right)=\left[\begin{array}{ll}
\widetilde{\mathcal{R}}^{x x}\left(C_{3(z)}\right) & \widetilde{\mathcal{R}}^{x y}\left(C_{3(z)}\right) \\
\widetilde{\mathcal{R}}^{y x}\left(C_{3(z)}\right) & \widetilde{\mathcal{R}}^{z z}\left(C_{3(z)}\right)
\end{array}\right]=\left[\begin{array}{cc}
-\frac{1}{2} & \frac{\sqrt{3}}{2} \\
-\frac{\sqrt{3}}{2} & -\frac{1}{2}
\end{array}\right]\left[\begin{array}{cc}
\mathcal{R}^{x x} & \mathcal{R}^{x y} \\
\mathcal{R}^{y x} & \mathcal{R}^{z z}
\end{array}\right]\left[\begin{array}{cc}
-\frac{1}{2} & -\frac{\sqrt{3}}{2} \\
\frac{\sqrt{3}}{2} & -\frac{1}{2}
\end{array}\right] .
$$

Then the Raman vertices of $\mathrm{E}_{2}$ symmetry species behave as

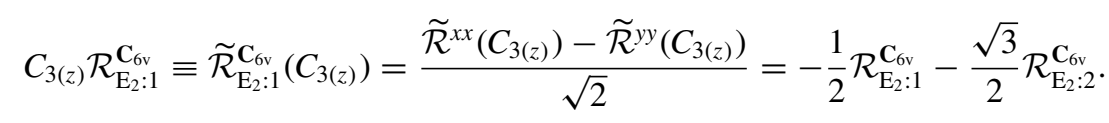


The Raman response of the Kitaev honeycomb QSL remains unchanged against the symmetry operation $C_{3(z)} \in \mathbf{C}_{6 \mathrm{v}}$,

$$
I_{\mathrm{E}_{2}: 1}^{\mathbf{C}_{6 v}}(\omega)=C_{3(z)} I_{\mathrm{E}_{2}: 1}^{\mathbf{C}_{6 \mathrm{v}}}(\omega)=\int_{-\infty}^{\infty} \frac{d t e^{i \omega t}}{2 \pi \hbar L}\left\langle 0\left|e^{\frac{i \mathscr{H} t}{\hbar}} \widetilde{\mathcal{R}}_{\mathrm{E}_{2}: 1}^{\mathbf{C}_{6 \mathrm{v}}}\left(C_{3(z)}\right) e^{-\frac{i \mathscr{H} t}{\hbar}} \widetilde{\mathcal{R}}_{\mathrm{E}_{2}: 1}^{\mathbf{C}_{6 \mathrm{v}}}\left(C_{3(z)}\right)\right| 0\right\rangle=\frac{1}{4} I_{\mathrm{E}_{2}: 1}^{\mathbf{C}_{6 \mathrm{v}}}(\omega)+\frac{3}{4} I_{\mathrm{E}_{2}: 2}^{\mathbf{C}_{6 \mathrm{v}}}(\omega),
$$

and therefore, we find that $I_{\mathrm{E}_{2}: 1}^{\mathbf{C}_{6 \mathrm{v}}}(\omega)=I_{\mathrm{E}_{2}: 2}^{\mathbf{C}_{6 \mathrm{v}}}(\omega)$. Next we consider rotating the $\mathbf{T}$ and $\mathbf{O}_{\mathrm{h}}$ polyhedra by $\frac{2 \pi}{3}$ about the [111] axis, which reads converting the Raman operator into

$$
C_{3(111)} \mathcal{R} \equiv\left[\begin{array}{lll}
\widetilde{\mathcal{R}}^{x x}\left(C_{3(111)}\right) & \widetilde{\mathcal{R}}^{x y}\left(C_{3(111)}\right) & \widetilde{\mathcal{R}}^{x z}\left(C_{3(111)}\right) \\
\widetilde{\mathcal{R}}^{y x}\left(C_{3(111)}\right) & \widetilde{\mathcal{R}}^{y y}\left(C_{3(111)}\right) & \widetilde{\mathcal{R}}^{y z}\left(C_{3(111)}\right) \\
\widetilde{\mathcal{R}}^{z x}\left(C_{3(111)}\right) & \widetilde{\mathcal{R}}^{z y}\left(C_{3(111)}\right) & \widetilde{\mathcal{R}}^{z z}\left(C_{3(111)}\right)
\end{array}\right]=\left[\begin{array}{lll}
0 & 1 & 0 \\
0 & 0 & 1 \\
1 & 0 & 0
\end{array}\right]\left[\begin{array}{ccc}
\mathcal{R}^{x x} & \mathcal{R}^{x y} & \mathcal{R}^{x z} \\
\mathcal{R}^{y x} & \mathcal{R}^{y y} & \mathcal{R}^{y z} \\
\mathcal{R}^{z x} & \mathcal{R}^{z y} & \mathcal{R}^{z z}
\end{array}\right]\left[\begin{array}{lll}
0 & 0 & 1 \\
1 & 0 & 0 \\
0 & 1 & 0
\end{array}\right] .
$$

They each have the two Raman-active symmetry species $\mathrm{E} / \mathrm{E}_{\mathrm{g}}$ and $\mathrm{T} / \mathrm{T}_{2 \mathrm{~g}}$, and the corresponding Raman vertices behave under the threefold rotation as

$$
\begin{aligned}
& C_{3(111)} \mathcal{R}_{\mathrm{E} / \mathrm{E}_{\mathrm{g}}: 1}^{\mathrm{T} / \mathbf{o}_{\mathrm{h}}} \equiv \widetilde{\mathcal{R}}_{\mathrm{E} / \mathrm{E}_{\mathrm{g}}: 1}^{\mathrm{T} / \mathbf{O}_{\mathrm{h}}}\left(C_{3(111)}\right)=\frac{2 \widetilde{\mathcal{R}}^{z z}\left(C_{3(111)}\right)-\widetilde{\mathcal{R}}^{x x}\left(C_{3(111)}\right)-\widetilde{\mathcal{R}}^{y y}\left(C_{3(111)}\right)}{\sqrt{6}}=-\frac{1}{2} \mathcal{R}_{\mathrm{E} / \mathrm{E}_{\mathrm{h}}: 1}^{\mathrm{T} / \mathbf{O}_{\mathrm{h}}}+\frac{\sqrt{3}}{2} \mathcal{R}_{\mathrm{E} / \mathrm{E}_{\mathrm{g}}: 2}^{\mathrm{T} / \mathbf{O}_{\mathrm{h}}}, \\
& C_{3(111)} \mathcal{R}_{\mathrm{T} / \mathrm{T}_{2 g}: 1}^{\mathrm{T} / \mathbf{O}_{\mathrm{h}}} \equiv \widetilde{\mathcal{R}}_{\mathrm{T} / \mathrm{T}_{2 g}: 1}^{\mathrm{T} / \mathbf{o}_{\mathrm{h}}}\left(C_{3(111)}\right)=\frac{\widetilde{\mathcal{R}}^{x y}\left(C_{3(111)}\right)+\widetilde{\mathcal{R}}^{y x}\left(C_{3(111)}\right)}{\sqrt{2}}=\mathcal{R}_{\mathrm{T} / \mathrm{T}_{2 g}: 2}^{\mathrm{T} / \mathbf{O}_{\mathrm{h}}}, \\
& C_{3(111)} \mathcal{R}_{\mathrm{T} / \mathrm{T}_{2 g}: 2}^{\mathrm{T} / \mathbf{o}_{\mathrm{h}}} \equiv \widetilde{\mathcal{R}}_{\mathrm{T} / \mathrm{T}_{2 g}: 2}^{\mathrm{T} / \mathbf{O}_{\mathrm{h}}}\left(C_{3(111)}\right)=\frac{\widetilde{\mathcal{R}}^{y z}\left(C_{3(111)}\right)+\widetilde{\mathcal{R}}^{z y}\left(C_{3(111)}\right)}{\sqrt{2}}=\mathcal{R}_{\mathrm{T} / \mathrm{T}_{2 g}:: 3}^{\mathrm{T} / \mathbf{o}_{\mathrm{h}}}, \\
& C_{3(111)} \mathcal{R}_{\mathrm{T} / \mathrm{T}_{2 g}: 3}^{\mathrm{T} / \mathbf{O}_{\mathrm{h}}} \equiv \widetilde{\mathcal{R}}_{\mathrm{T} / \mathrm{T}_{2 g}: 3}^{\mathrm{T} / \mathbf{O}_{\mathrm{h}}}\left(C_{3(111)}\right)=\frac{\widetilde{\mathcal{R}}^{z x}\left(C_{3(111)}\right)+\widetilde{\mathcal{R}}^{x z}\left(C_{3(111)}\right)}{\sqrt{2}}=\mathcal{R}_{\mathrm{T} / \mathrm{T}_{2 g}: 1}^{\mathrm{T} / \mathbf{O}_{\mathrm{h}}} .
\end{aligned}
$$

The Raman responses of these Kitaev polyhedral QSLs are invariant under their common symmetry operation $C_{3(111)}$,

$$
\begin{aligned}
& I_{\mathrm{E} / \mathrm{E}_{\mathrm{g}}: 1}^{\mathbf{T} / \mathbf{O}_{\mathrm{h}}}(\omega)=C_{3(111)} I_{\mathrm{E} / \mathrm{E}_{\mathrm{g}}: 1}^{\mathbf{T} / \mathbf{O}_{\mathrm{h}}}(\omega)=\frac{1}{4} I_{\mathrm{E} / \mathrm{E}_{\mathrm{g}}: 1}^{\mathbf{T} / \mathbf{O}_{\mathrm{h}}}(\omega)+\frac{3}{4} I_{\mathrm{E} / \mathrm{E}_{\mathrm{g}}: 2}^{\mathbf{T} / \mathbf{o}_{\mathrm{h}}}(\omega), \\
& I_{\mathrm{T} / \mathrm{T}_{2 g}: 1}^{\mathbf{T} / \mathbf{O}_{\mathrm{h}}}(\omega)=C_{3(111)} I_{\mathrm{T} / \mathrm{T}_{2 \mathrm{~h}}: 1}^{\mathbf{T} / \mathbf{O}_{\mathrm{h}}}(\omega)=I_{\mathrm{T} / \mathrm{T}_{2 \mathrm{~h}}: 2}^{\mathbf{T} / \mathbf{O}_{\mathrm{h}}}(\omega), \\
& I_{\mathrm{T} / \mathrm{T}_{2 \mathrm{~h}}: 2}^{\mathbf{T} / \mathbf{O}_{\mathrm{h}}}(\omega)=C_{3(111)} I_{\mathrm{T} / \mathrm{T}_{2 \mathrm{~g}}: 2}^{\mathbf{T} / \mathbf{O}_{\mathrm{h}}}(\omega)=I_{\mathrm{T} / \mathrm{T}_{2 \mathrm{~g}}: 3}^{\mathbf{T} / \mathbf{O}_{\mathrm{h}}}(\omega), \\
& I_{\mathrm{T} / \mathrm{T}_{2 \mathrm{~h}}: 3}^{\mathbf{T} / \mathbf{O}_{\mathrm{h}}}(\omega)=C_{3(111)} I_{\mathrm{T} / \mathrm{T}_{2 \mathrm{~g}}: 3}^{\mathbf{T} / \mathbf{O}_{\mathrm{h}}}(\omega)=I_{\mathrm{T} / \mathrm{T}_{2 \mathrm{~h}}: 1}^{\mathbf{T} / \mathbf{O}_{\mathrm{h}}}(\omega),
\end{aligned}
$$

and therefore, we find that $I_{\mathrm{E} / \mathrm{E}_{\mathrm{g}}: 1}^{\mathbf{T} / \mathbf{h}_{\mathrm{h}}}(\omega)=I_{\mathrm{E} / \mathrm{E}_{\mathrm{g}}: 2}^{\mathbf{T} / \mathbf{O}_{\mathrm{h}}}(\omega)$ and $I_{\mathrm{T} / \mathrm{T}_{2 \mathrm{~g}}: 1}^{\mathbf{T} / \mathbf{O}_{\mathrm{h}}}(\omega)=I_{\mathrm{T} / \mathrm{T}_{2 \mathrm{~g}}: 2}^{\mathbf{T} / \mathbf{O}_{\mathrm{h}}}(\omega)=I_{\mathrm{T} / \mathrm{T}_{2 \mathrm{~g}}: 3}^{\mathbf{T} / \mathbf{O}_{\mathrm{h}}}(\omega)$. For the Raman-active H symmetry species of the Kitaev dodecahedral QSL as well, we can similarly find the spectral degeneracy $I_{\mathrm{H}: 1}^{\mathbf{I}}(\omega)=I_{\mathrm{H}: 2}^{\mathrm{I}}(\omega)=I_{\mathrm{H}: 3}^{\mathbf{I}}(\omega)=$ $I_{\mathrm{H}: 4}^{\mathbf{I}}(\omega)=I_{\mathrm{H}: 5}^{\mathbf{I}}(\omega)$.

Now that Eq. (D6) reduces to

$$
I(\omega)=\sum_{i}^{\prime} \sum_{\mu=1}^{d_{\Xi_{i}}^{\mathbf{P}}}\left(E_{\Xi_{i}: \mu}^{\mathbf{P}}\right)^{2} I_{\Xi_{i}: \mu}^{\mathbf{P}}(\omega)=\sum_{i}^{\prime} I_{\Xi_{i}: 1}^{\mathbf{P}}(\omega) \sum_{\mu=1}^{d_{\Xi_{i}}^{\mathbf{P}}}\left(E_{\Xi_{i}: \mu}^{\mathbf{P}}\right)^{2},
$$

the number of Raman-active modes possible in the lattice geometry is most decisive of whether and how the scattering intensity depends on the light polarization. In Eq. (D16), we have

$$
\sum_{\mu=1}^{2}\left(E_{\mathrm{E}_{2}: \mu}^{\mathbf{C}_{6 \mathrm{v}}}\right)^{2}=\frac{1}{2} \sin ^{2} \vartheta_{\text {in }} \sin ^{2} \vartheta_{\mathrm{sc}}
$$

for the two-dimensional honeycomb lattice,

$$
\begin{aligned}
\sum_{\mu=1}^{5}\left(E_{\mathrm{H}: \mu}^{\mathbf{I}}\right)^{2}= & \frac{1}{6}\left[2 \cos \vartheta_{\text {in }} \cos \vartheta_{\mathrm{sc}}-\sin \vartheta_{\text {in }} \sin \vartheta_{\mathrm{sc}} \cos \left(\varphi_{\text {in }}-\varphi_{\mathrm{sc}}\right)\right]^{2}+\frac{1}{2}\left[\sin \vartheta_{\text {in }} \sin \vartheta_{\mathrm{sc}} \cos \left(\varphi_{\text {in }}+\varphi_{\mathrm{sc}}\right)\right]^{2} \\
& +\frac{1}{2}\left[\sin \vartheta_{\text {in }} \sin \vartheta_{\mathrm{sc}} \sin \left(\varphi_{\text {in }}+\varphi_{\mathrm{sc}}\right)\right]^{2}+\frac{1}{2}\left(\sin \vartheta_{\text {in }} \sin \varphi_{\text {in }} \cos \vartheta_{\mathrm{sc}}+\cos \vartheta_{\text {in }} \sin \vartheta_{\mathrm{sc}} \sin \varphi_{\mathrm{sc}}\right)^{2} \\
& +\frac{1}{2}\left(\cos \vartheta_{\text {in }} \sin \vartheta_{\mathrm{sc}} \cos \varphi_{\mathrm{sc}}+\sin \vartheta_{\text {in }} \cos \varphi_{\text {in }} \cos \vartheta_{\mathrm{sc}}\right)^{2}
\end{aligned}
$$


for the dodecahedral lattice, and

$$
\begin{aligned}
\sum_{\mu=1}^{2}\left(E_{\mathrm{E}: \mu}^{\mathbf{T}}\right)^{2}=\sum_{\mu=1}^{2}\left(E_{\mathrm{E}_{\mathrm{g}}: \mu}^{\mathbf{O}_{\mathrm{h}}}\right)^{2}= & \frac{1}{6}\left[2 \cos \vartheta_{\text {in }} \cos \vartheta_{\mathrm{sc}}-\sin \vartheta_{\text {in }} \sin \vartheta_{\mathrm{sc}} \cos \left(\varphi_{\mathrm{in}}-\varphi_{\mathrm{sc}}\right)\right]^{2}+\frac{1}{2}\left[\sin \vartheta_{\text {in }} \sin \vartheta_{\mathrm{sc}} \cos \left(\varphi_{\mathrm{in}}+\varphi_{\mathrm{sc}}\right)\right]^{2}, \\
\sum_{\mu=1}^{3}\left(E_{\mathrm{T}: \mu}^{\mathbf{T}}\right)^{2}=\sum_{\mu=1}^{3}\left(E_{\mathrm{T}_{2 \mathrm{~g}}: \mu}^{\mathbf{O}_{\mathrm{h}}}\right)^{2}= & \frac{1}{2}\left[\sin \vartheta_{\text {in }} \sin \vartheta_{\mathrm{sc}} \sin \left(\varphi_{\text {in }}+\varphi_{\mathrm{sc}}\right)\right]^{2}+\frac{1}{2}\left(\sin \vartheta_{\text {in }} \sin \varphi_{\text {in }} \cos \vartheta_{\mathrm{sc}}+\cos \vartheta_{\text {in }} \sin \vartheta_{\mathrm{sc}} \sin \varphi_{\mathrm{sc}}\right)^{2} \\
& +\frac{1}{2}\left(\cos \vartheta_{\text {in }} \sin \vartheta_{\mathrm{sc}} \cos \varphi_{\mathrm{sc}}+\sin \vartheta_{\text {in }} \cos \varphi_{\text {in }} \cos \vartheta_{\mathrm{sc}}\right)^{2}
\end{aligned}
$$

for the truncated tetrahedral and octahedral lattices, respectively. For the honeycomb lattice, we are interested only in the polarization vectors parallel to the plane,

$$
\left.\sum_{\mu=1}^{2}\left(E_{\mathrm{E}_{2}: \mu}^{\mathbf{C}_{\mathrm{vi}}}\right)^{2}\right|_{\vartheta_{\mathrm{in}}=\vartheta_{\mathrm{sc}}=\frac{\pi}{2}}=\frac{1}{2},
$$

and find no polarization dependence of the Raman response within the LF scheme. For the dodecahedral lattice, even if we restrict the polarization vectors to the $x y$ plane, the Raman response still exhibits weak polarization dependence even within the LF scheme,

$$
\left.\sum_{\mu=1}^{5}\left(E_{\mathrm{H}: \mu}^{\mathbf{I}}\right)^{2}\right|_{\vartheta_{\mathrm{in}}=\vartheta_{\mathrm{sc}}=\frac{\pi}{2}}=\frac{1}{6} \cos ^{2}\left(\varphi_{\mathrm{in}}-\varphi_{\mathrm{sc}}\right)+\frac{1}{2},
$$

i.e., the spectra peak exactly the same but weigh differently according to the light polarization. For the truncated tetrahedral and octahedral lattices, even if we consider the Raman scattering within the LF scheme and restrict the polarization vectors to the $x y$ plane, we have two Raman-active symmetry species to find strong polarization dependence of the spectra,

$$
\begin{aligned}
& \left.\sum_{\mu=1}^{2}\left(E_{\mathrm{E}: \mu}^{\mathbf{T}}\right)^{2}\right|_{\vartheta_{\mathrm{in}=}=\vartheta_{\mathrm{sc}}=\frac{\pi}{2}}=\left.\sum_{\mu=1}^{2}\left(E_{\mathrm{E}_{\mathrm{g}}: \mu}^{\mathbf{O}_{\mathrm{h}}}\right)^{2}\right|_{\vartheta_{\mathrm{in}}=\vartheta_{\mathrm{sc}}=\frac{\pi}{2}}=\frac{1}{6} \cos ^{2}\left(\varphi_{\mathrm{in}}-\varphi_{\mathrm{sc}}\right)+\frac{1}{2} \cos ^{2}\left(\varphi_{\mathrm{in}}+\varphi_{\mathrm{sc}}\right), \\
& \left.\sum_{\mu=1}^{3}\left(E_{\mathrm{T}: \mu}^{\mathbf{T}}\right)^{2}\right|_{\vartheta_{\mathrm{in}}=\vartheta_{\mathrm{sc}}=\frac{\pi}{2}}=\left.\sum_{\mu=1}^{3}\left(E_{\mathrm{T}_{2 \mathrm{~g}}: \mu}^{\mathbf{O}_{\mathrm{h}}}\right)^{2}\right|_{\vartheta_{\mathrm{in}}=\vartheta_{\mathrm{sc}}=\frac{\pi}{2}}=\frac{1}{2} \sin ^{2}\left(\varphi_{\mathrm{in}}+\varphi_{\mathrm{sc}}\right),
\end{aligned}
$$

i.e., spectra peak and weigh differently according to the light polarization. Note in this context that we do not have any accidental degeneracy, i.e., neither does $I_{\mathrm{E}: 1}^{\mathrm{T}}(\omega)$ equal $I_{\mathrm{T}: 1}^{\mathrm{T}}(\omega)$ nor does $I_{\mathrm{E}_{\mathrm{g}}: 1}^{\mathbf{O}_{\mathrm{h}}}(\omega)$ equal $I_{\mathrm{T}_{2 \mathrm{~g}}: 1}^{\mathbf{O}_{\mathrm{h}}}(\omega)$.

[1] A. Kitaev, Ann. Phys. (NY) 321, 2 (2006).

[2] L. Savary and L. Balents, Rep. Prog. Phys. 80, 016502 (2017).

[3] Y. Zhou, K. Kanoda, and T.-K. Ng, Rev. Mod. Phys. 89, 025003 (2017).

[4] J. Knolle and R. Moessner, Annu. Rev. Condens. Matter Phys. 10, 451 (2019).

[5] Y. Motome and J. Nasu, J. Phys. Soc. Jpn. 89, 012002 (2020).

[6] G. Jackeli and G. Khaliullin, Phys. Rev. Lett. 102, 017205 (2009).

[7] Y. Singh and P. Gegenwart, Phys. Rev. B 82, 064412 (2010).

[8] Y. Singh, S. Manni, J. Reuther, T. Berlijn, R. Thomale, W. Ku, S. Trebst, and P. Gegenwart, Phys. Rev. Lett. 108, 127203 (2012).

[9] K. Kitagawa, T. Takayama, Y. Matsumoto, A. Kato, R. Takano, Y. Kishimoto, S. Bette, R. Dinnebier, G. Jackeli, and H. Takagi, Nature 554, 341 (2018).

[10] K. W. Plumb, J. P. Clancy, L. J. Sandilands, V. V. Shankar, Y. F. Hu, K. S. Burch, H.-Y. Kee, and Y.-J. Kim, Phys. Rev. B 90, 041112(R) (2014).
[11] J. Chaloupka, G. Jackeli, and G. Khaliullin, Phys. Rev. Lett. 105, 027204 (2010).

[12] J. Chaloupka, G. Jackeli, and G. Khaliullin, Phys. Rev. Lett. 110, 097204 (2013).

[13] K. Slagle, W. Choi, L. E. Chern, and Y. B. Kim, Phys. Rev. B 97, 115159 (2018).

[14] H. Tomishige, J. Nasu, and A. Koga, Phys. Rev. B 97, 094403 (2018).

[15] U. F. P. Seifert, J. Gritsch, E. Wagner, D. G. Joshi, W. Brenig, M. Vojta, and K. P. Schmidt, Phys. Rev. B 98, 155101 (2018).

[16] H. Tomishige, J. Nasu, and A. Koga, Phys. Rev. B 99, 174424 (2019).

[17] J. G. Rau, E. K.-H. Lee, and H.-Y. Kee, Phys. Rev. Lett. 112, 077204 (2014).

[18] V. M. Katukuri, S. Nishimoto, V. Yushankhai, A. Stoyanova, H. Kandpal, S. Choi, R. Coldea, I. Rousochatzakis, L. Hozoi, and J. van den Brink, New J. Phys. 16, 013056 (2014).

[19] Y. Yamaji, Y. Nomura, M. Kurita, R. Arita, and M. Imada, Phys. Rev. Lett. 113, 107201 (2014). 
[20] T. Suzuki, T. Yamada, Y. Yamaji, and S. Suga, Phys. Rev. B 92, 184411 (2015).

[21] Y. Yamaji, T. Suzuki, T. Yamada, S. Suga, N. Kawashima, and M. Imada, Phys. Rev. B 93, 174425 (2016).

[22] M. Gohlke, G. Wachtel, Y. Yamaji, F. Pollmann, and Y. B. Kim, Phys. Rev. B 97, 075126 (2018).

[23] J. Knolle, S. Bhattacharjee, and R. Moessner, Phys. Rev. B 97, 134432 (2018).

[24] J. Knolle, G.-W. Chern, D. L. Kovrizhin, R. Moessner, and N. B. Perkins, Phys. Rev. Lett. 113, 187201 (2014).

[25] J. Nasu, J. Knolle, D. L. Kovrizhin, Y. Motome, and R. Moessner, Nat. Phys. 12, 912 (2016).

[26] B. Perreault, J. Knolle, N. B. Perkins, and F. J. Burnell, Phys. Rev. B 94, 060408(R) (2016).

[27] B. Perreault, J. Knolle, N. B. Perkins, and F. J. Burnell, Phys. Rev. B 94, 104427 (2016).

[28] B. Perreault, S. Rachel, F. J. Burnell, and J. Knolle, Phys. Rev. B 95, 184429 (2017).

[29] I. Rousochatzakis, S. Kourtis, J. Knolle, R. Moessner, and N. B. Perkins, Phys. Rev. B 100, 045117 (2019).

[30] S. K. Choi, R. Coldea, A. N. Kolmogorov, T. Lancaster, I. I. Mazin, S. J. Blundell, P. G. Radaelli, Y. Singh, P. Gegenwart, K. R. Choi, S.-W. Cheong, P. J. Baker, C. Stock, and J. Taylor, Phys. Rev. Lett. 108, 127204 (2012).

[31] A. Banerjee, C. A. Bridges, J.-Q. Yan, A. A. Aczel, L. Li, M. B. Stone, G. E. Granroth, M. D. Lumsden, Y. Yiu, J. Knolle, S. Bhattacharjee, D. L. Kovrizhin, R. Moessner, D. A. Tennant, D. G. Mandrus, and S. E. Nagler, Nat. Mater. 15, 733 (2016).

[32] A. Banerjee, J. Yan, J. Knolle, C. A. Bridges, M. B. Stone, M. D. Lumsden, D. G. Mandrus, D. A. Tennant, R. Moessner, and S. E. Nagler, Science 356, 1055 (2017).

[33] S.-H. Do, S.-Y. Park, J. Yoshitake, J. Nasu, Y. Motome, Y. S. Kwon, D. T. Adroja, D. J. Voneshen, K. Kim, T.-H. Jang, J.-H. Park, K.-Y. Choi, and S. Ji, Nat. Phys. 13, 1079 (2017).

[34] L. J. Sandilands, Y. Tian, K. W. Plumb, Y.-J. Kim, and K. S. Burch, Phys. Rev. Lett. 114, 147201 (2015).

[35] S. Yamamoto and T. Kimura, J. Phys.: Conf. Ser. 1220, 012003 (2019).

[36] T. Takayama, A. Kato, R. Dinnebier, J. Nuss, H. Kono, L. S. I. Veiga, G. Fabbris, D. Haskel, and H. Takagi, Phys. Rev. Lett. 114, 077202 (2015).

[37] K. A. Modic, T. E. Smidt, I. Kimchi, N. P. Breznay, A. Biffin, S. Choi, R. D. Johnson, R. Coldea, P. Watkins-Curry, G. T. McCandless, J. Y. Chan, F. Gandara, Z. Islam, A. Vishwanath, A. Shekhter, R. D. McDonald, and J. G. Analytis, Nat. Commun. 5, 4203 (2014).

[38] S. Mandal and N. Surendran, Phys. Rev. B 79, 024426 (2009).

[39] J. Nasu, M. Udagawa, and Y. Motome, Phys. Rev. Lett. 113, 197205 (2014).

[40] I. Kimchi, J. G. Analytis, and A. Vishwanath, Phys. Rev. B 90, 205126 (2014).

[41] M. Hermanns and S. Trebst, Phys. Rev. B 89, 235102 (2014).

[42] K. O'Brien, M. Hermanns, and S. Trebst, Phys. Rev. B 93, 085101 (2016).

[43] Y. Kato, Y. Kamiya, J. Nasu, and Y. Motome, Phys. Rev. B 96, 174409 (2017).

[44] A. Smith, J. Knolle, D. L. Kovrizhin, J. T. Chalker, and R. Moessner, Phys. Rev. B 93, 235146 (2016).
[45] K. Suzuki and S. Yamamoto, J. Phys.: Conf. Ser. 1220, 012046 (2019).

[46] M. Thakurathi, K. Sengupta, and D. Sen, Phys. Rev. B 89, 235434 (2014).

[47] W. DeGottardi, D. Sen, and S. Vishveshwara, New J. Phys. 13, 065028 (2011).

[48] F. L. Pedrocchi, S. Chesi, S. Gangadharaiah, and D. Loss, Phys. Rev. B 86, 205412 (2012).

[49] P. Mellado, O. Petrova, and O. Tchernyshyov, Phys. Rev. B 91, 041103(R) (2015).

[50] X.-G. Wen, Phys. Rev. B 65, 165113 (2002).

[51] F. Wang and A. Vishwanath, Phys. Rev. B 74, 174423 (2006).

[52] H. Yao and S. A. Kivelson, Phys. Rev. Lett. 99, 247203 (2007).

[53] P. A. Fleury and R. Loudon, Phys. Rev. 166, 514 (1968).

[54] B. S. Shastry and B. I. Shraiman, Phys. Rev. Lett. 65, 1068 (1990).

[55] B. S. Shastry and B. I. Shraiman, Int. J. Mod. Phys. B 5, 365 (1991).

[56] O. Petrova, P. Mellado, and O. Tchernyshyov, Phys. Rev. B 90, 134404 (2014).

[57] H. Yao, S.-C. Zhang, and S. A. Kivelson, Phys. Rev. Lett. 102, 217202 (2009).

[58] F. L. Pedrocchi, S. Chesi, and D. Loss, Phys. Rev. B 84, 165414 (2011).

[59] F. Zschocke and M. Vojta, Phys. Rev. B 92, 014403 (2015).

[60] M. Udagawa, Phys. Rev. B 98, 220404(R) (2018).

[61] M. S. Dresselhaus, G. Dresselhaus, and A. Jorio, Group Theory: Application to the Physics of Condensed Matter (Springer, Berlin, 2008).

[62] B. Perreault, J. Knolle, N. B. Perkins, and F. J. Burnell, Phys. Rev. B 92, 094439 (2015).

[63] When we identify the ground state with Eq. (9) without specifying its gauge fields $\left|\left\{u_{\langle m, n\rangle_{\lambda}}\right\}\right\rangle_{0(r)}$, it belongs to $\mathbf{P}$ rather than $\widetilde{\mathbf{P}}$. If we recognize the ground state by its gauge fields, $|0\rangle \equiv\left|\left\{n_{k}\right\}\right\rangle_{0} \otimes\left|\left\{u_{\langle m, n\rangle_{\lambda}}\right\}\right\rangle_{0(0)}$, for instance, with $\left|\left\{n_{k}\right\}\right\rangle_{\kappa} \otimes$ $\left|\left\{u_{\langle m, n\rangle_{\lambda}}\right\}\right\rangle_{q(r)}$ being the $\kappa$ th spinon state $\left(\kappa=0, \ldots, 2^{\frac{L}{2}}-1\right)$ against the $q(r)$ th bond configuration $\left[q(r)=2^{L-1} q+r ; q=\right.$ $\left.0, \ldots, 2^{\frac{L}{2}+1}-1, r=0, \ldots, 2^{L-1}-1\right],|0\rangle$ chosen in the augmented Hilbert space is no longer invariant under every symmetry operation of $\mathbf{P}$ but belongs to its $\mathbb{Z}_{2}$-gauge extension $\widetilde{\mathbf{P}}$. See Appendix A for further details.

[64] T. P. Devereaux and R. Hackl, Rev. Mod. Phys. 79, 175 (2007).

[65] O. Cépas, J. O. Haerter, and C. Lhuillier, Phys. Rev. B 77, 172406 (2008).

[66] W.-H. Ko, Z.-X. Liu, T.-K. Ng, and P. A. Lee, Phys. Rev. B 81, 024414 (2010).

[67] N. Perkins and W. Brenig, Phys. Rev. B 77, 174412 (2008).

[68] J. Knolle, D. L. Kovrizhin, J. T. Chalker, and R. Moessner, Phys. Rev. Lett. 112, 207203 (2014).

[69] J. Knolle, D. L. Kovrizhin, J. T. Chalker, and R. Moessner, Phys. Rev. B 92, 115127 (2015).

[70] A. Trautman, in Clifford Analysis and Its Applications, edited by F. Brackx, J. S. R. Chisholm, and V. Souček (Springer, Dordrecht, Netherlands, 2001), p. 377.

[71] S. Yamamoto, Phys. Rev. B 63, 125124 (2001).

[72] S. Yamamoto and T. Kimura, J. Phys. Soc. Jpn. 89, 063701 (2020). 